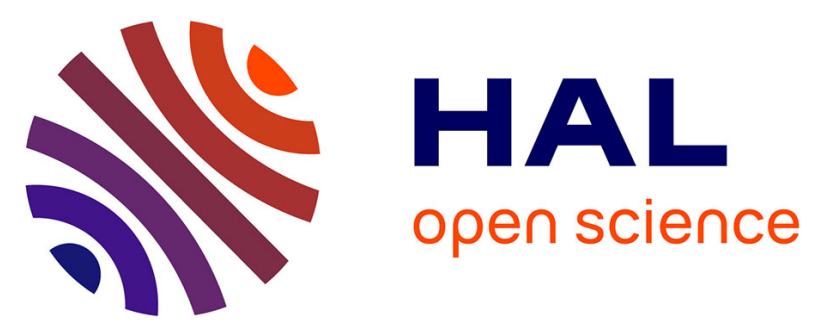

\title{
Biodiversity across the Guadalupian-Lopingian Boundary: first results on the ostracod (Crustacea) fauna, Chaotian section (Sichuan Province, South China)
}

Sindbad Zazzali, Sylvie Crasquin, Jean-François Deconinck, Qinglai Feng

\section{- To cite this version:}

Sindbad Zazzali, Sylvie Crasquin, Jean-François Deconinck, Qinglai Feng. Biodiversity across the Guadalupian-Lopingian Boundary: first results on the ostracod (Crustacea) fauna, Chaotian section (Sichuan Province, South China). Geodiversitas, 2015, 37 (3), pp.283-313. 10.5252/g2015n3a1. hal-01260450

\section{HAL Id: hal-01260450 \\ https: / hal.sorbonne-universite.fr/hal-01260450}

Submitted on 22 Jan 2016

HAL is a multi-disciplinary open access archive for the deposit and dissemination of scientific research documents, whether they are published or not. The documents may come from teaching and research institutions in France or abroad, or from public or private research centers.
L'archive ouverte pluridisciplinaire HAL, est destinée au dépôt et à la diffusion de documents scientifiques de niveau recherche, publiés ou non, émanant des établissements d'enseignement et de recherche français ou étrangers, des laboratoires publics ou privés.

\section{(ㄷ)(i)}

Distributed under a Creative Commons Attribution| 4.0 International License 


\title{
Biodiversity across the Guadalupian-Lopingian Boundary: first results on the ostracod (Crustacea) fauna, Chaotian section (Sichuan Province, South China)
}

\author{
Sindbad ZAZZALI \\ Sylvie CRASQUIN \\ CR2P UMR 7207, Sorbonne Universités - CNRS - MNHN - UPMC Université Paris 06, \\ T. 46-56, E.5, case 104, 4 place Jussieu, F-75252 Paris cedex 05 (France) \\ sindbad.zazzali@upmc.fr \\ sylvie.crasquin@upmc.fr
}

Jean-François DECONINCK Université de Bourgogne, UMR 6282 CNRS Biogéosciences, 6 boulevard Gabriel, F-21000 Dijon (France) jean-francois.deconinck@u-bourgogne.fr

Qinglai FENG State Key Laboratory of Geological Process and Mineral Resources, University of Geosciences, No. 388, Lumo Road, Wuhan 430074 (People's Republic of China) qinglaifeng@cug.edu.cn

Published on 25 September 2015

urn:Isid:zoobank.org:pub:5F708564-E50F-4452-ACF4-06157C83A841

Zazzali S., Crasquin S., Deconinck J.-F. \& Feng Q. 2015. - Biodiversity across the Guadalupian-Lopingian Boundary: first results on the ostracod (Crustacea) fauna, Chaotian section (Sichuan Province, South China). Geodiversitas 37 (3): 283-313. http://dx.doi.org/10.5252/g2015n3a1

ABSTRACT

The Middle Permian-Late Permian boundary (Guadalupian-Lopingian boundary, GLB) interval is characterised by important faunal assemblage changes. This extinction-turnover episode is considered by some authors to be the first step of the end-Permian biodiversity drop. The forty-five meters thickness of sediment encompassing the GLB in Chaotian section (Sichuan Province, South China) was sampled and processed for ostracod study. This study presents the first analysis of ostracod faunas in the GLB interval. A total of 154 species belonging to 29 genera are identified. Three species are described as new: Bairdia chaotianensis Zazzali, n. sp., Microcheilinella wujiapingensis Zazzali,

KEY WORDS Crustacea, Ostracods South China, Biodiversity, Middle-Late Permian, Guadalupian-Lopingian boundary, Capitanian-

Wuchiapingian, new species. n. sp., Microcheilinella pagodaensis Zazzali, n. sp. All the ostracods discovered in the section belong to shallow marine taxa. So these results are not consistent with previous interpretations (lagoonal environment or deep water setting) based on other evidences. Abundance and diversity present a rapid and noticeable decline in the Early Capitanian. Recovery is then recorded about three meters above the GLB. At specific level, a 93\% extinction rate and a 96\% turnover rate are recorded at the GLB. Moreover, Palaeocopida, straight dorsal border ostracods known to progressively disappear from the Late Permian to the basal Middle-Triassic, are here less abundant and diversified after the GLB. This could reflect the first step of their disappearance at the end of the Palaeozoic. 


\author{
MOTS CLÉS \\ Crustacea, \\ Ostracodes, \\ Chine du Sud, \\ Biodiversité, \\ Permien moyen \\ Limite Guadalupien- \\ Lopingien, \\ Capitanien- \\ Wuchiapingien, \\ espèces nouvelles.
}

\begin{abstract}
RÉSUMÉ
Biodiversité à la limite Guadalupien-Lopingien: premiers résultats sur la faune d'ostracodes, coupe de Chaotian (province du Sichuan, Chine du Sud).

L'intervalle de temps bordant la limite Permien moyen-Permien supérieur (limite GuadalupienLopingien, GLB) est caractérisé par d'importantes modifications d'assemblages. Cet épisode d'extinctions-renouvellement est considéré par certains auteurs comme la première étape du déclin de la biodiversité à la fin du Permien. Les échantillons prélevés sur les quarante-cinq mètres encadrant la GLB à Chaotian (province du Sichuan, Chine du Sud) ont été traités pour l'étude des ostracodes. Les travaux présentés ici constituent la toute première analyse des faunes d'ostracodes dans l'intervalle de temps bordant la GLB. 154 espèces appartenant à 29 genres sont identifiées. Trois nouvelles espèces sont décrites: Bairdia chaotianensis Zazzali, n. sp., Microcheilinella wujiapingensis Zazzali, n. sp., Microcheilinella pagodaensis Zazzali, n. sp. Les ostracodes retrouvés au sein de la coupe appartiennent tous à des taxons marins peu profonds. Ces résultats ne sont pas en accord avec les précédentes interprétations (milieu lagunaire ou milieu très profond) basées sur l'étude de divers autres paramètres et taxons. L'abondance et la diversité déclinent rapidement et significativement au Capitanien inférieur. La récupération est ensuite enregistrée environ trois mètres au-dessus de la GLB. Au niveau spécifique, un taux d'extinction de $93 \%$ et un taux de renouvellement de $96 \%$ sont enregistrés à GLB. De plus, les Palaeocopida, connus pour disparaitre progressivement du Permien supérieur à la base du Trias moyen, sont ici moins abondants et diversifiés après la GLB. Cela pourrait constituer la première étape de leur disparition à la fin du Paléozoïque.
\end{abstract}

\section{INTRODUCTION}

Late Permian (Lopingian) and Early Triassic times are characterised by major global environmental changes and events leading to the most important mass extinction of the all Phanerozoic. Another biological event was evidenced 7.7My before the PTB (Permian-Triassic Boundary) in the upper part of the Guadalupian (Middle Permian), before the Guadalupian-Lopingian Boundary (GLB). It was first demonstrated by a turnover in foraminifera and particularly amongst fusulinids (Stanley \& Yang 1994; Jin et al. 1994). Stanley \& Yang (1994) estimated that $71 \%$ of marine species already disappeared at the end of the Capitanian (Late Guadalupian). More recent studies reveal that the extinctions already occurred in the Middle Capitanian within Jinogondolella altudaensis conodont Zone, significantly below the GLB (Fig. 1) (Bond et al. 2010b). This marine crisis affected also corals, bryozoans, brachiopods, bivalves, calcareous algae and ammonoids (e.g. Jin et al. 1995; Wang \& Sugiyama 2000; Isozaki \& Aljinovic 2009; Shen \& Shi 2009; Bond et al. 2010b). Bond et al. (2010a) highlighted this crisis also on land, with extinction of 24\% of plants in South China and 56\% in North China. Several geological phenomena have been suggested to be at the origin of this biodiversity drop: regression (Isozaki et al. 2008; Wignall et al. 2009), volcanism (Emeishan flood basalt Province, Isozaki et al. 2007, 2008; He et al. 2010), methane release (Thompson et al. 2001; Retallack \& Jahren 2008) and climatic changes (Veizer et al. 2000; Isozaki et al. 2007; Isozaki 2009).
The PTB and GLB biodiversity drops are considered by some authors as a double-phased extinction event in the Late Palaeozoic (Yin et al. 2007). Some others favor a gradual decrease in diversity from the Wordian (Middle Guadalupian) up to the end of the Permian (Clapham et al. 2009). This paper presents the first results on ostracod fauna at the GLB based on the study of the reference Chaotian section (Sichuan Province, P. R. China; Fig. 2).

\section{GEOLOGICAL SETTING}

During the Late Palaeozoic-Early Mesozoic period, the South China block (SCB) was located on the eastern part of Pangaea, in equatorial position (Fig. 3A). It was bathed by the Palaeotethys Ocean to the West and by the Panthalassa mega-ocean to the East (Scotese \& Langford 1995). The SCB was at this time in a $90^{\circ}$ counter clockwise position with regard to the present day location (Fig. 3B). Middle Permian and earliest Triassic (Induan) carbonate sediments are well-exposed along the two banks of the Jialingjiang River, north of Chaotian (north of Guangyuan, NE Sichuan, Figs 2 ; 4$)$. The Chaotian section $\left(30^{\circ} 23.713 \mathrm{~N}-106^{\circ} 49.615 \mathrm{E}\right)$ mainly consists of marine shelf carbonates deposited on the Palaeo-Tethyan side of South China block (Isozaki et al. 2008). Late Guadalupian (Capitanian) sediments of the Maokou Fm consist of thick massive calcareous beds with occasional flint layers notably at base of the section. This informally designed "Unit 1" of Lai et al. (2008) and "Main Limestone" of Isozaki et al. (2008) was recently named 
"Limestone Unit" by Saitoh et al. (2013a). It is divided in three subunits, L1 to L3, dated from Wordian to Early Capitanian by conodonts (Isozaki et al. 2008; Lai et al. 2008; Saitoh et al. 2013a). We consider here only the upper part of the last subunit, L3, which is Early Capitanian in age (Jinogondolella postserrata conodont Zone, Fig. 1) and is mainly composed of packstones with marine fossils, including calcareous algae, foraminifers and ostracods (Isozaki et al. 2008).

The upper part of the Maokou Fm is represented by a 11 m series called "Unit 2" by Lai et al. (2008), "Uppermost Member" by Isozaki et al. (2008) and "Mudstone Unit" by Saitoh et al. (2013a, b). It is composed of an alternation of thinly bedded black calcareous mudstones, black cherts, black siliceous mudstones, dark grey limestones and dark grey dolostones. Saitoh et al. (2013a) subdivided this "Mudstone Unit" in two subunits. The M1 lower carbonate-rich one contains conodonts, fragmented brachiopods, small foraminifera and ostracods. It is Early-Middle Capitanian in age (J. shannoni conodont Zone and Pseudoalbaillella longtanensis-P. globosa radiolarian assemblage in Saitoh et al. 2013a). The M2 upper subunit, is dominated by black calcareous mudstones yielding radiolarians, ammonoids and gastropods in some chert layers. The age of M2 is not clearly constrained and it is possible that the Late Capitanian is missing (Saitoh et al. 2013a). The "Mudstone Unit" is capped by the Wangpo Bed (Fig. 4), a two-meter-thick deeply weathered air-fall ash bed called "G-L Boundary acidic tuff” (Isozaki et al. 2004), and termed Xuanwei Fm in South China (He et al. 2007) where volcanoclastic deposits are common at the GLB (Wignall et al. 2009; Bond et al. 2010a). The volcanic origin of the Wangpo Bed was recently challenged by $\mathrm{He}$ et al. (2010) who suggest that it corresponds to a clastic deposit originating from the erosion of volcanic rocks from the Emeishan Large Igneous Province. Recent analysis of clay mineral assemblages of the Wangpo Bed (Deconinck et al. 2014) precises that they are composed of volcanic particles directly deposited in marine environment reworked with volcanic material first deposited on continent. The base of the Wangpo Bed is directly dated c. $260 \pm 4 \mathrm{My}$ by U-Pb dating of zircons (He et al. 2007). The Wangpo Bed is considered by Saitoh et al. $(2013 \mathrm{a}, \mathrm{b})$ to belong to the Wujiaping Fm but it is a distinct "unit" which clearly separates the Maokou Fm from the overlying strata.

The Wujiaping Fm begins by a black argillaceous horizon, called "black level", also referred as coaly mudstone with plant debris (Isozaki et al. 2008). The facies drastically change after this interval. Indeed, the overlying sediments are dominantly composed of bioclastic packstones with many foraminifera (but with less diverse populations of fusulinids than in the Maokou Fm), calcareous algae, siliceous sponge spicules, ostracods and brachiopods (Isozaki et al. 2008; Lai et al. 2008; Saitoh et al. 2013a). Numerous centimetric to decimetric yellowish to buff-coloured tuff layers are intercalated in this formation with an upward increasing frequency. We pay attention here to the

\begin{tabular}{|c|c|c|c|c|}
\hline \multirow{8}{*}{ 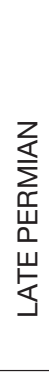 } & \multirow{8}{*}{ 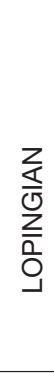 } & & Conodont Zones & \\
\hline & & \multirow{7}{*}{ 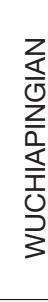 } & Clarkina orientalis & \multirow{7}{*}{ 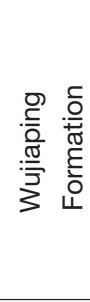 } \\
\hline & & & C. transcausasia & \\
\hline & & & C. guiangyuanensis & \\
\hline & & & C. leveni & \\
\hline & & & C. asymmetrica & \\
\hline & & & C. dukouensis & \\
\hline & & & C. postbitteri postbitteri & \\
\hline \multirow{7}{*}{ 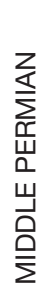 } & \multirow{7}{*}{ 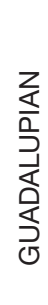 } & \multirow{7}{*}{ 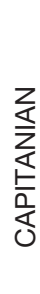 } & C. postbitteri hongshuensis & \multirow{7}{*}{ 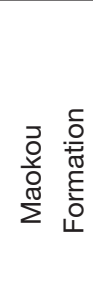 } \\
\hline & & & Jinogondolella granti & \\
\hline & & & J. xuanhanensis & \\
\hline & & & J. prexuanhanensis & \\
\hline & & & J. altudaensis & \\
\hline & & & J. shannoni & \\
\hline & & & J. posteserrata & \\
\hline
\end{tabular}

FIG. 1. - Middle-Late Permian boundary conodont Zones and South China formations (modified after Mei \& Henderson 2001; Jin et al. 2006).

27 first meters of the Wujiaping Fm. The presence of Codonofusiella-Reichelina small fusuline assemblage in the first levels of this formation, allows Isozaki et al. (2008) and Saitoh et al. (2013a) to locate the GLB at the base of the bioclastic limestones.

There is an agreement about the palaeoenvironmental interpretation of "Limestone Unit" of the Maokou Fm and lower part of the Wujiaping Fm which are both deposited on oxic shelf in the euphotic zone. The "black level", at the base of the Wujiaping Fm, is interpreted as near-shore to continental environment sediments deposited after a major regressive event at the end of the Capitanian (Isozaki et al. 2008; Lai et al. 2008; Saitoh et al. 2013a, b). Similar facies are widespread in South China and are referred to the Heshan Fm. These coal seams were deposited in swamps developed on or adjacent to tidal-flats (Shao et al. 1998, 2003). The significance of the "Mudstone Unit" is more controversial. Isozaki et al. (2008) and Saitoh et al. (2013a, b) consider that it represents a two-step deepening (first one during M1 subunit and second one during M2 subunit) up to oxygen-depleted slope/basin environment in the disphotic zone. These authors consider that there is a very sharp sea-level drop during or after the deposition of the Wangpo Bed. According to Lai et al. (2008), Unit 2 (="Mudstone Unit") was deposited in a shallow environment close to emergence.

\section{MATERIAL AND METHODS}

Forty-six samples, distributed through the 45 m thick sequence flanking the GLB (Fig. 4), have been processed for ostracod analysis. The "Mudstone Unit" presents facies (mudstones and cherts) unfavourable for ostracod preservation and release. The M1 and M2 subunit are mainly silicified. 


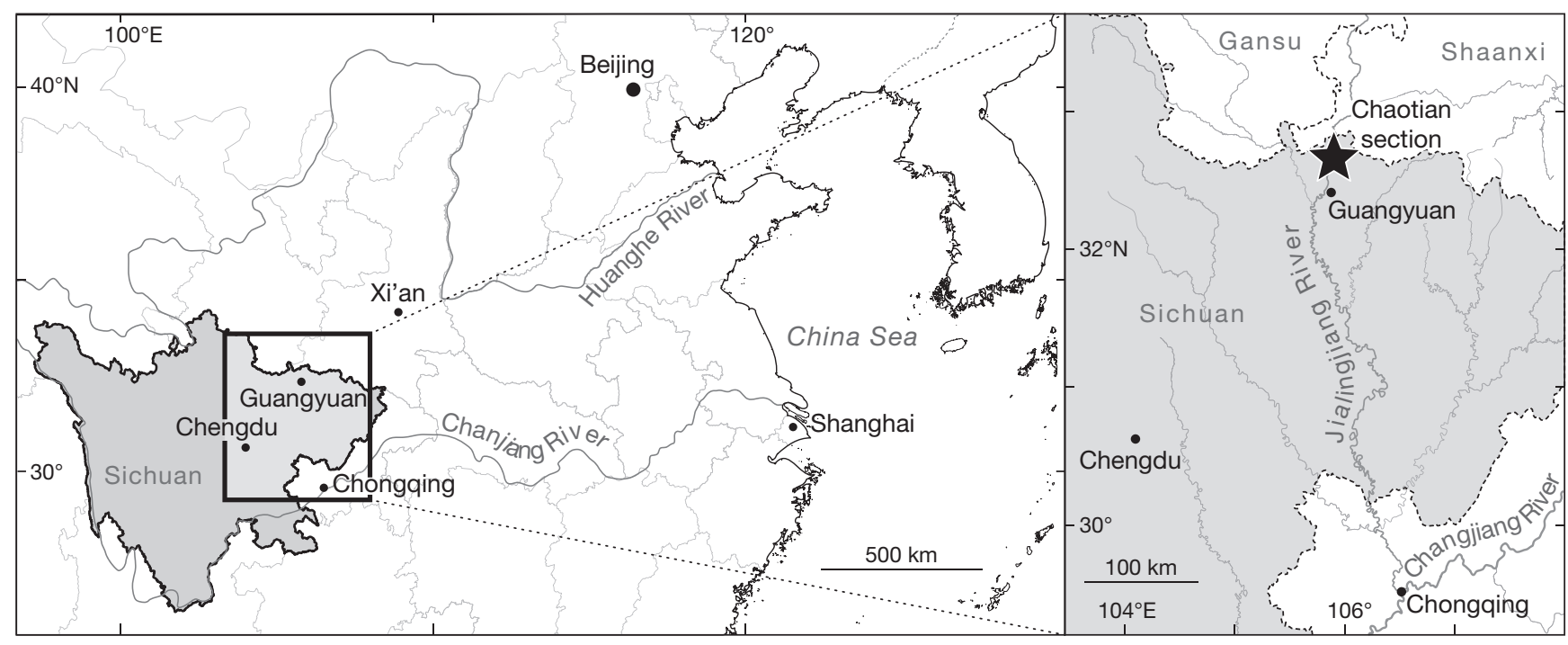

FIG. 2. - Location map of Chaotian section, Sichuan, P. R. China (modified after Isozaki et al. 2007).

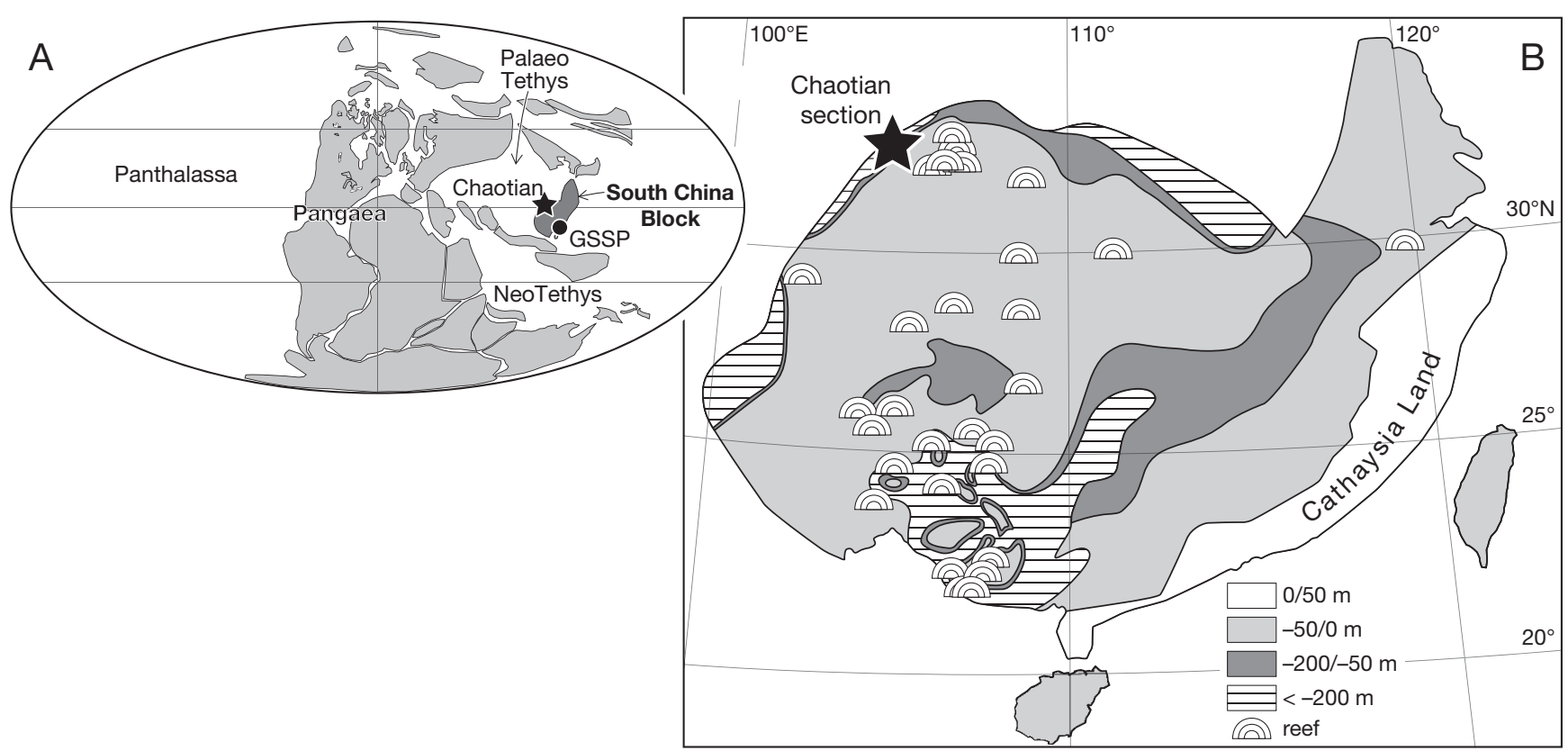

FIG. 3. - A, Palaeogeographic map of the Middle-Late Permian (modified after Golonka 2002); B, distribution of Capitanian palaeoenvironments in South China (map in present day latitudes, modified from Wang \& Jin 2000).

Only the lower part of the M1 subunit, more calcareous, was sampled (11CHAO153 to $11 \mathrm{CHAO} 158)$. Due to the facies, the Wangpo Bed composed of ashes and the coaly "black level" were not taken in consideration in this study (11CHAO159 to 11CHAO162).

The extraction of calcareous ostracod carapaces from calcareous rocks is achieved by hot acetolysis (Lethiers \& CrasquinSoleau 1988; Crasquin-Soleau et al. 2005). An average of 15 grams from the sediment fine fraction (between 530 and $140 \mu \mathrm{m})$ has been studied for each sample. Six samples are barren, five from the Maokou Fm (11CHAO152, L3 subunit; 11CHAO153/154/155/157, M1 subunit) and one from the Wujiaping Fm (11CHAO78). From the 40 productive samples (Fig. 4), three yielded only unidentifiable specimens (11CHAO75/76/77).

A total of 154 species belonging to 29 genera are identified and figured (Table 1; Figs 5-17). Three new species are described, 11 species are formally recognized and one is compared to a previously described one. With regard to the poor preservation and/or the low number of specimens, all the other species are presented in open nomenclature but are nevertheless figured (Figs 5-17).

All specimens are deposited in the Université Pierre et Marie Curie, Paris (UPMC) collection (numbers: P6Mxxxx). 
TABLE 1. - Stratigraphic distribution of Capitanian-Wuchiapingien ostracods in Chaotian section. Abundances are indicated by numerical values (number of specimens)

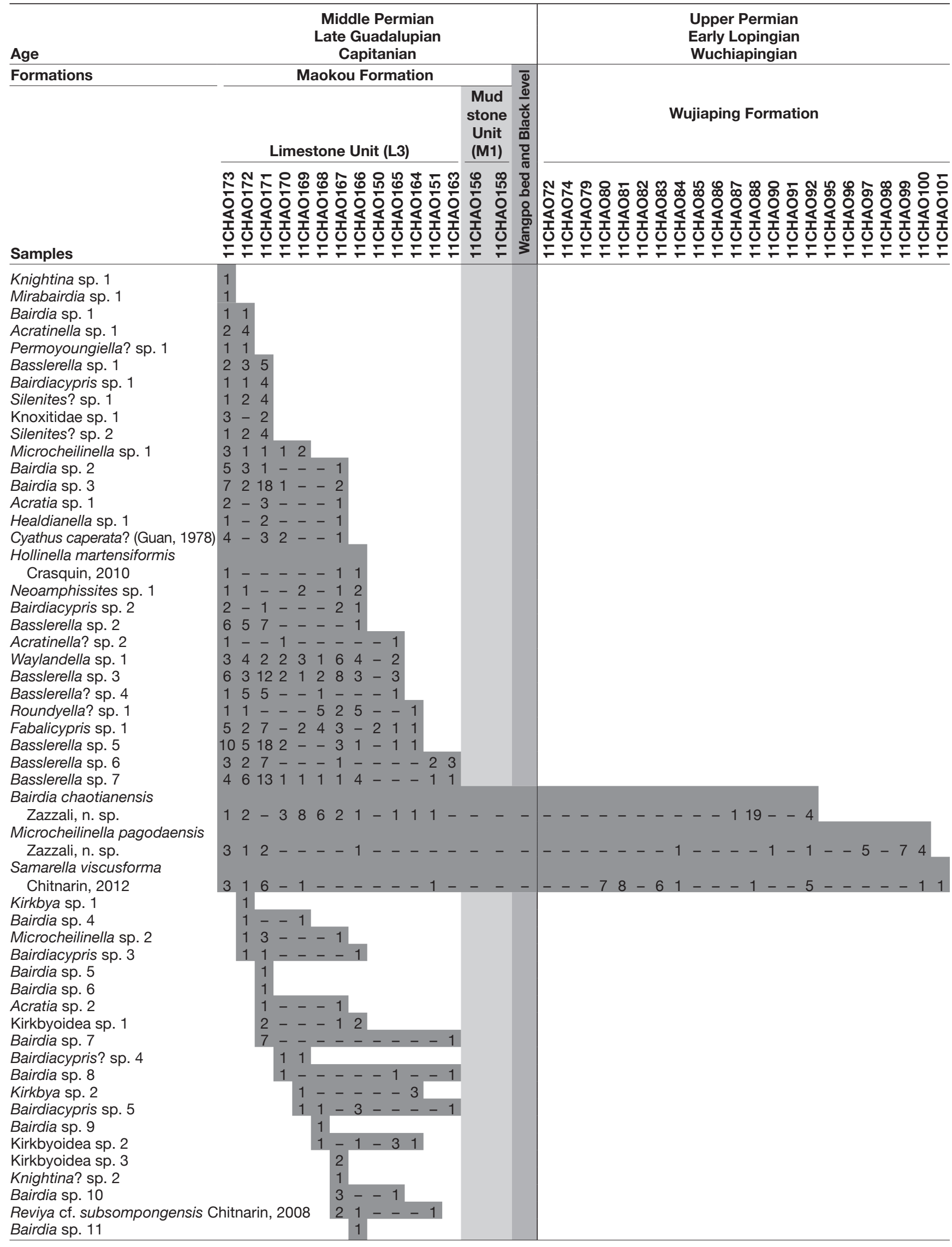


TABLE 1. - Continuation.

\begin{tabular}{|c|c|c|c|c|}
\hline \multirow{2}{*}{$\begin{array}{l}\text { Age } \\
\text { Formations }\end{array}$} & \multicolumn{3}{|l|}{$\begin{array}{l}\text { Middle Permian } \\
\text { Late Guadalupian } \\
\text { Capitanian }\end{array}$} & $\begin{array}{l}\text { Upper Permian } \\
\text { Early Lopingian } \\
\text { Wuchiapingian }\end{array}$ \\
\hline & \multicolumn{2}{|l|}{ Maokou Formation } & $\Phi$ & \multirow[b]{2}{*}{ Wujiaping Formation } \\
\hline \multirow[b]{2}{*}{ Samples } & Limestone Unit (L3) & $\begin{array}{c}\text { Mud } \\
\text { stone } \\
\text { Unit } \\
\text { (M1) } \\
\end{array}$ & $\begin{array}{l}\frac{3}{0} \\
\frac{0}{0} \\
\frac{\pi}{0} \\
0 \\
\end{array}$ & \\
\hline & 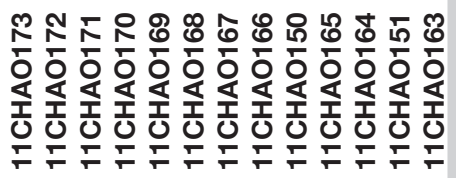 & 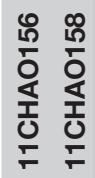 & $\begin{array}{l}\frac{0}{8} \\
\frac{0}{0} \\
\frac{0}{2} \\
\frac{\pi}{5} \\
3\end{array}$ & 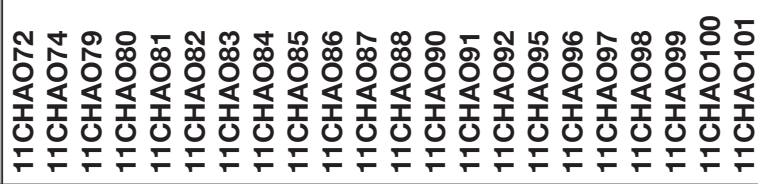 \\
\hline \multicolumn{5}{|c|}{ Kirkbya sp. 3} \\
\hline \multirow{2}{*}{\multicolumn{5}{|c|}{$\begin{array}{l}\text { Sulcella suprapermiana? Kozur, } 1985 \\
\text { Kindlella sp. } 1\end{array}$}} \\
\hline & & & & \\
\hline \multicolumn{5}{|l|}{ Roundyella sp. 2} \\
\hline \multicolumn{5}{|l|}{ Bairdia sp. 12} \\
\hline Microcheilinella? sp. 3 & & 8 & & \\
\hline \multicolumn{5}{|l|}{ Knoxiella sp. 1} \\
\hline \multirow{2}{*}{\multicolumn{5}{|c|}{$\begin{array}{l}\text { Kirkbya sp. } 4 \\
\text { Hollinella sp. } 1\end{array}$}} \\
\hline & & & & \\
\hline \multicolumn{5}{|l|}{ Cyathus elliptica? Shi, 1987} \\
\hline \multicolumn{5}{|l|}{ Acratia sp. 3} \\
\hline \multicolumn{5}{|c|}{$\begin{array}{l}\text { Geffenina posterodorsospina? Chitnarin, } 2012 \\
\text { Knoxiella sp. }\end{array}$} \\
\hline Fabalicypris sp. 2 & & & & $711-2$ \\
\hline Microcheilinella sp. 5 & & & & $1------1---4$ \\
\hline Acratia sp. 4 & & & & 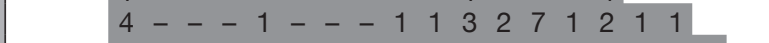 \\
\hline Microcheilinella sp. 6 & & & & $1----1----1-2--2$ \\
\hline Microcheilinella rectodorsata? & Forel, 2010 & & & $12--1----1--21$ \\
\hline Basslerella sp. 8 & & & & $4---1--7-13445122121$ \\
\hline Basslerella sp. 9 & & & & $1511212----121-1132$ \\
\hline Bairdiacypris sp. 6 & & & & $1331----1----1$ \\
\hline Bairdia sp. 13 & & & & 3 \\
\hline Microcheilinella sp. 7 & & & & \\
\hline Acratia nagyvisnyoensis? Forel & , 2013 & & & $5--1$ \\
\hline Bairdia sp. 14 & & & & $31---1$ \\
\hline Microcheilinella sp. 8 & & & & $212----2-1$ \\
\hline Microcheilinella sp. 9 & & & & $2-----1----1-1$ \\
\hline Kegelites sp. 1 & & & & $11-------1-1-1$ \\
\hline Microcheilinella sp. 10 & & & & $1---2---1---1$ \\
\hline Bairdia sp. 15 & & & & $11-1-1---513-2297$ \\
\hline Bairdia sp. 16 & & & & $1--1--111--1-11$ \\
\hline Bairdiacypris sp. 7 & & & & $2---51-4-2--114$ \\
\hline Acratinella sp. 3 & & & & $1--\ldots--112211-32$ \\
\hline Acratia recurvata? Cordell, 195 & & & & $1--1---1--7-3111$ \\
\hline Silenites? sp. 3 & & & & $1-\cdots-\cdots-1-2331$ \\
\hline Bairdia sp. 17 & & & & 1 \\
\hline Petasobairdia sp. 1 & & & & $1----9$ \\
\hline Acratinella sp. 4 & & & & $-\ldots---11---1$ \\
\hline Bairdia sp. 18 & & & & 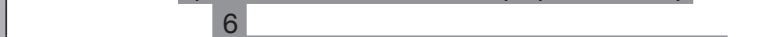 \\
\hline Fabalicypris sp. 3 & & & & $1---1----1-1--2$ \\
\hline Basslerella sp. 10 & & & & $11-31-122-1-43$ \\
\hline Petasobairdia sp. 2 & & & & $112-\ldots---1$ \\
\hline Microcheilinella sp. 11 & & & & $11---6-5134$ \\
\hline Bairdia sp. 19 & & & & $3--1-\ldots-1$ \\
\hline Bairdia sp. 20 & & & & 291 \\
\hline Silenites? sp. 4 & & & & 24 \\
\hline Bairdia sp. 21 & & & & $3---4$ \\
\hline Bairdia sp. 22 & & & & $12-1-12$ \\
\hline Microcheilinella sp. 12 & & & & $1 \overline{1}---1-1$ \\
\hline Bairdia sp. 23 & & & & $2--2-411$ \\
\hline Bairdia sp. 24 & & & & $1--2--6252$ \\
\hline Bairdia sp. 25 & & & & $1-1--3-2--1$ \\
\hline Bairdia sp. 26 & & & & $32--2--1145$ \\
\hline Bairdia sp. 27 & & & & $2--11--1-42$ \\
\hline Bairdiacypris sp. 8 & & & & $\begin{array}{lllllllll}1 & 6 & 1 & 1 & -6 & 1 & 3 & 3 & 2\end{array}$ \\
\hline
\end{tabular}


TABLE 1. - Continuation

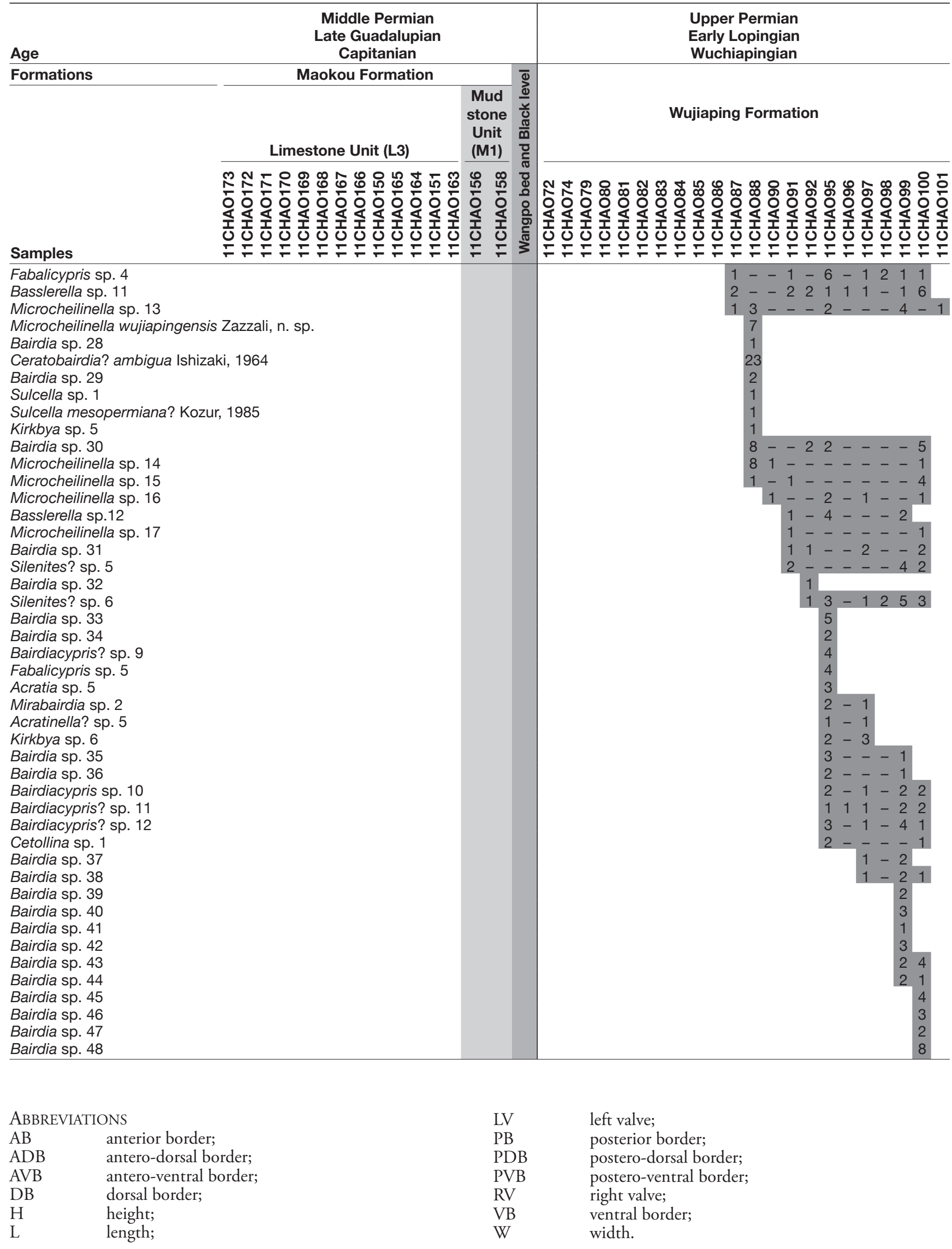




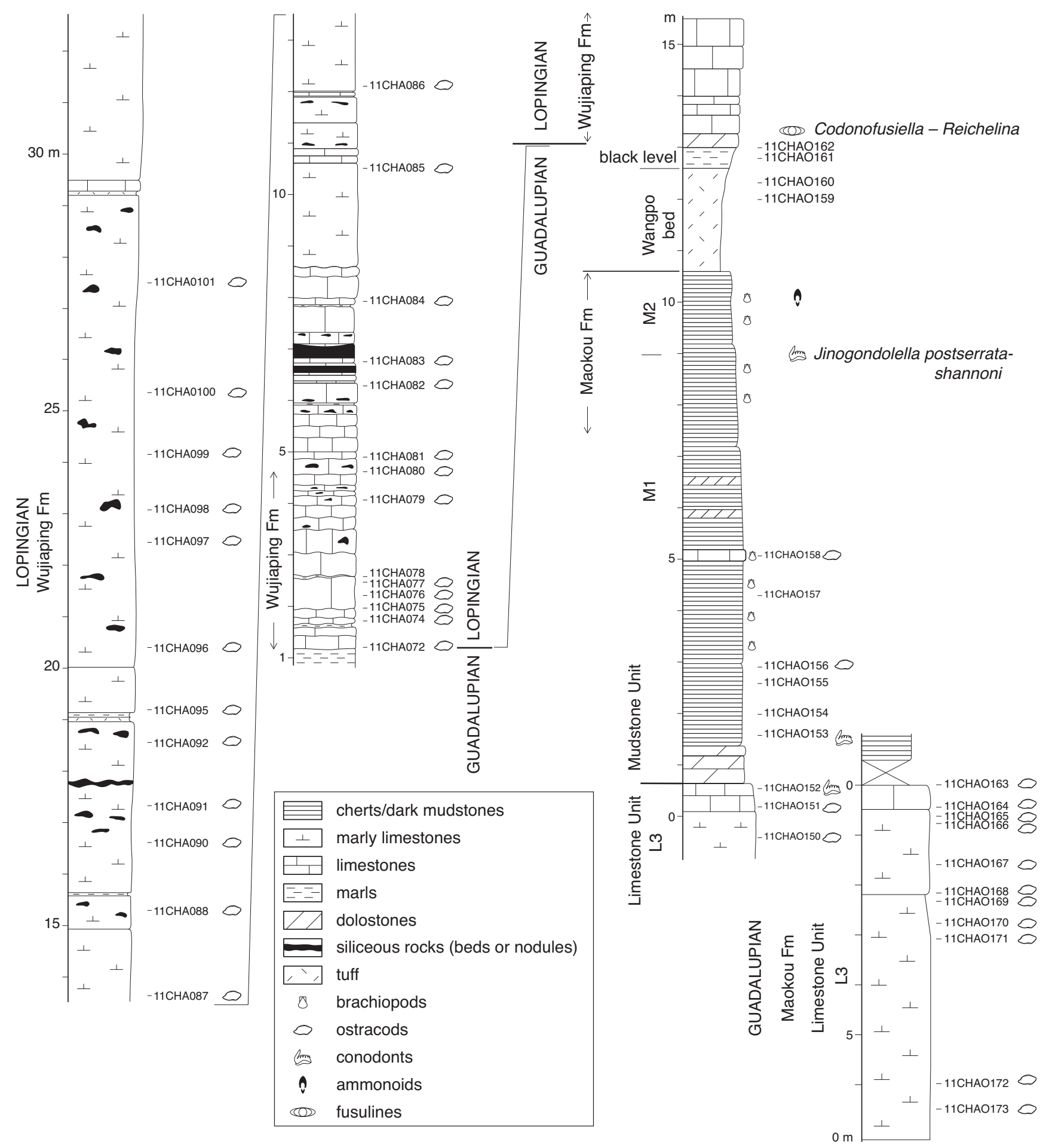

FIG. 4. - Stratigraphic column of the Chaotian section, South China, with location of studied samples. Ammonoid occurrences from Zhao et al. (1978), conodont and fusuline occurrences from Isozaki et al. (2008) and Lai et al. (2008).

\section{SYSTEMATIC PALEONTOLOGY}

The classification adopted here follows Moore (1961) and Horne et al. (2002).
Order PODOCOPIDA Müller, 1894

Suborder PODOCOPINA Sars, 1866

Superfamily BAIRDIOIDEA Sars, 1887

Family ACRATIIDAE Gründel, 1962

$$
\text { Genus Acratia Delo, } 1930
$$

Class OSTRACODA Latreille, 1806

Subclass PODOCOPA Müller, 1894

TYPe SPECIES. - Acratia typica Delo, 1930 by original designation. 


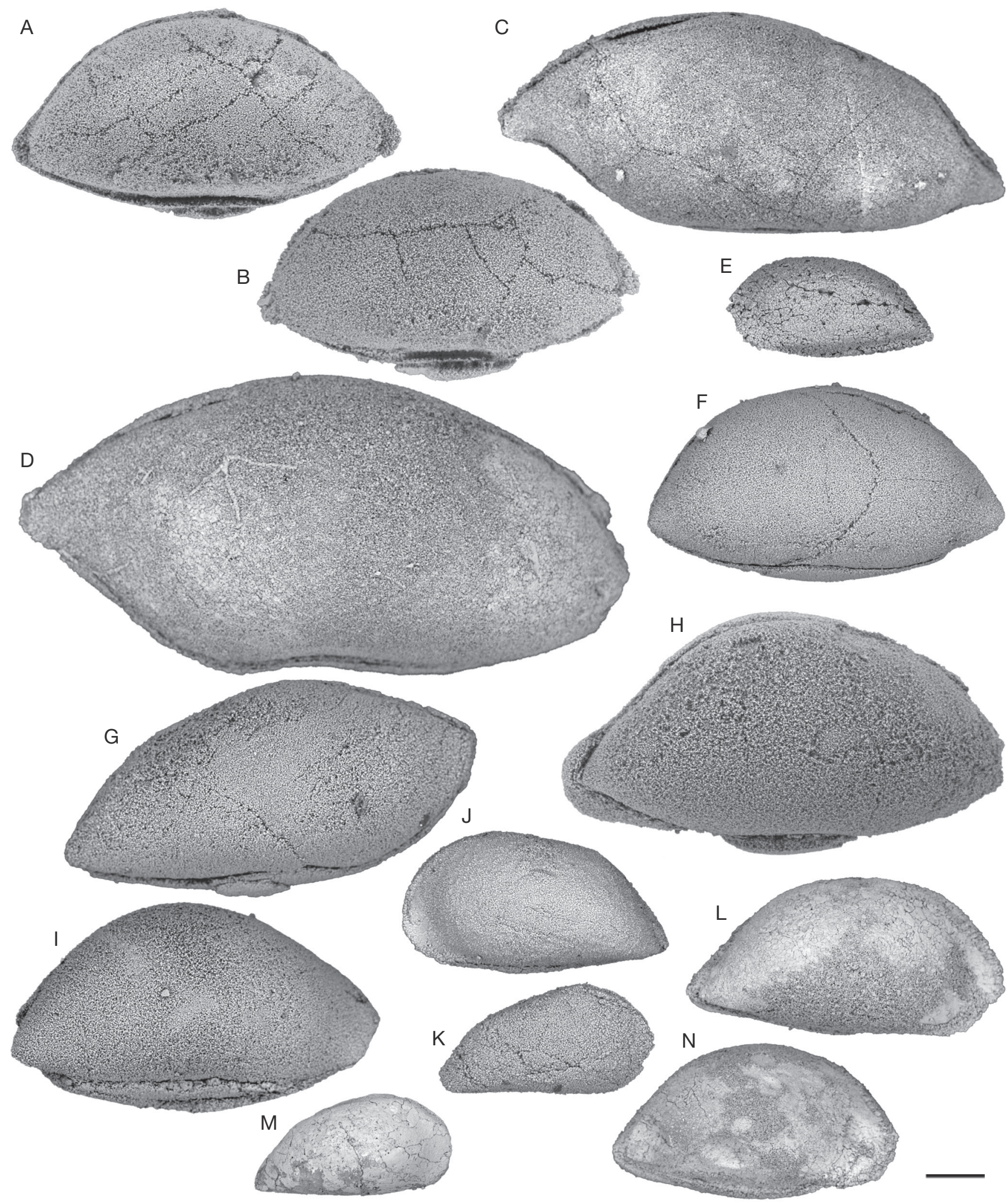

FIG. 5. - Ostracods from Guadalupian-Lopingian boundary interval in South China: A, B, Acratia nagyvisnyoensis? Forel, 2013; A, right lateral view, P6M3596, 11CHAO84; B, right lateral view, P6M3597, 11CHAO81; C, D, Acratia recurvata? Cordell, 1952; C, left lateral view, P6M3598, 11CHAO81; D, left lateral view, P6M3599, 11CHAO99; E, Acratia sp. 1, right lateral view, P6M3600, sample 11CHAO171; F. Acratia sp. 2, right lateral view, P6M3601, sample 11CHAO167; G, Acratia sp. 3, right lateral view, P6M3602, sample 11CHAO95; H, Acratia sp. 4, left lateral view, P6M3603, sample 11CHAO80; I, Acratia sp. 5, right lateral view, P6M3604, sample 11CHAO95; J, Acratinella sp. 1, left lateral view, P6M3605, sample 11CHAO173; K, Acratinella? sp. 2, right lateral view, P6M3606, sample 11CHAO173; L, Acratinella sp. 3, right lateral view, P6M3607, sample 11CHAO99; M, Acratinella sp. 4, right lateral view, P6M3608, sample 11CHAO99; N, Acratinella? sp. 5, right lateral view, P6M3609, sample 11CHAO97. All photographs represent complete carapaces. Scale bar: $100 \mu \mathrm{m}$. 
Acratia nagyvisnyoensis? Forel, 2013

(Fig. 5A, B)

Acratia nagyvisnyoensis Forel in Forel et al., 2013: 204, fig. 10K-O.

MATERIAL EXAMINED. - Four complete carapaces and two broken carapaces.

DimENSIONS. - $\mathrm{L}=629-682 \mu \mathrm{m} ; \mathrm{H}=308-373 \mu \mathrm{m}$.

OCCuRRENCES. - Bükk Mountains, Hungary, Late Permian (Forel et al. 2013); ?Chaotian section, Sichuan Province, China. Wujiaping Formation, basal Wuchiapingian, Late Permian (this study, samples: 11CHAO81, 11CHAO84, see Table 1).

\section{REMARKS}

Our specimens are very similar to $A$. nagyvisnyoensis Forel, 2013 from the Late Permian of Hungary (Forel et al. 2013) but differ in having longer carapaces and a less arched DB.

\section{Acratia recurvata? Cordell, 1952}

(Fig. 5C, D)

Acratia recurvata Cordell, 1952: 81, 82, pl. 20, fig. 65. - Shi \& Chen 2002: 81, 82, pl. 20, figs 19-24.

Material eXamined. - Three complete carapaces and 13 broken carapaces.

Dimensions. $-\mathrm{L}=499-1197 \mu \mathrm{m} ; \mathrm{H}=244-500 \mu \mathrm{m}$.

OCCURREnCES. - Oread Formation, Missouri, USA, Late Carboniferous (Cordell 1952); Guangxi, China, Late Permian (Shi \& Chen 2002); ?Chaotian section, Sichuan Province, China, Wujiaping Formation, basal Wuchiapingian, Late Permian (this study, samples: 11CHAO81, 11CHAO85, 11CHAO90, 11CHAO95, 11CHAO97, 11CHAO98, 11CHAO99, 11CHAO100, see Table 1).

\section{REMARKS}

Our specimens are very similar to A. recurvata Cordell, 1952 from the Late Pennsylvanian (Late Carboniferous) of Missouri (USA; Cordell 1952) and Late Permian of South China (Shi \& Chen 2002) but differ in having a more concave AVB, a VB slightly concave at mid-L and the PDB connection with $\mathrm{DB}$ forming a more or less marked angle.

Family BAIRDIIDAE Sars, 1887

Genus Bairdia McCoy, 1844

TyPe SPECIES. - Bairdia curta McCoy, 1844 subsequently designated by Ulrich \& Bassler (1923: 320).

Bairdia chaotianensis Zazzali, n. sp. (Fig. 6D, E; 18)

TyPe MATERial. - Holotype: one complete carapace (P6M3613; Fig. 6D); paratype: one complete carapace (P6M3614; Fig. 6E).

Etymology. - From the Chaotian section, Type locality.

MATERIAL EXAMINED. - 24 complete carapaces and 35 isolated valves.
Dimensions. $-\mathrm{L}=694-1160 \mu \mathrm{m} ; \mathrm{H}=322-631 \mu \mathrm{m}$. Holotype: $\mathrm{L}=1050 \mu \mathrm{m} ; \mathrm{H}=557 \mu \mathrm{m}$. Paratype: $\mathrm{L}=914 \mu \mathrm{m} ; \mathrm{H}=459 \mu \mathrm{m}$ (Fig. 18).

TYPE HORIZON. — Sample 11CHAO172, "Limestone Unit" (L3), Maokou Formation, Middle Capitanian, Middle Permian.

TYPE LOCALITY. - Chaotian section $\left(30^{\circ} 23.713 \mathrm{~N}-106^{\circ} 49.615 \mathrm{E}\right)$, Sichuan Province, P. R. China.

Occurrences. - Chaotian section, Sichuan Province, China, Maokou and Wujiaping Formations, Middle Capitanian-basal Wuchiapingian, Middle-Late Permian (samples: 11CHAO173, 11CHAO 172, 11CHAO 170, 11CHAO169, 11CHAO 168, 11CHAO 167, 11CHAO 166, 11CHAO 165, 11CHAO 164, 11CHAO151, 11CHAO87, 11CHAO88, 11CHAO92, see Table 1).

Diagnosis. - A species of Bairdia with elongated carapace, AB and $\mathrm{BP}$ with small radius of curvature, hinge line located below DB; both valves present a more or less expressed shoulder with a crest at the top, parallel to the hinge line.

\section{DESCRIPTION}

Overlap: absent at DB, narrow to absent at $\mathrm{AB}, \mathrm{PB}$ and $\mathrm{PVB}$, narrow at $\mathrm{AVB}$ and distinct at $\mathrm{ADB}, \mathrm{PDB}$ and VB.

$\mathrm{DB}$ broadly convex at right lateral view. Hinge line located below DB; presence of more or less expressed shoulder at DB of both valves with a crest at the top, parallel to the hinge line; shoulder and crest extend from $\mathrm{DB}$ to PDB at RV and from $\mathrm{ADB}$ to $\mathrm{PDB}$ at $\mathrm{LV}$.

$\mathrm{RV}$ : ADB significantly concave at its median part. AB with small radius of curvature. $\mathrm{AB}$ maximum convexity slightly above mid-H. AVB gently convex to nearly straight. VB faintly curved (convex) to nearly straight. PVB in the lengthening of $\mathrm{VB}$. PB with a smaller radius of curvature than anterior one. PB pointed near or slightly below mid-H. PDB more slightly concave than anterior one.

LV: ADB more slightly concave the RV one. AB with small radius of curvature. $\mathrm{AB}$ maximum convexity at mid-H. AVB gently convex to nearly straight. VB faintly curved (convex) to nearly straight. PVB gently curved. PB with a smaller radius of curvature than anterior one. $\mathrm{PB}$ pointed near or slightly below mid-H. PDB more slightly concave than RV one.

Greatest L slightly above mid-H. Greatest H slightly anterior to mid-L.

In dorsal view, lateral outlines asymmetrically convex, with a maximum of $\mathrm{W}$ at mid-L and mid-H. RV slightly to significantly wider than $\mathrm{LV}$.

$\mathrm{L} / \mathrm{H}=0.5$.

REMARKS

Within Wuchiapingian specimens some intraspecific variations are observed. Some specimens present slightly higher valves, wider carapaces and, at LV more prominent DB shoulder and crest than at RV. More or less faint angles could be observed at RV between DB and upper half of PDB and ADB. Some differences could also be noticed between Capitanian and Wuchiapingian specimens. Capitanian ones show more rounded valves, with a RV significantly wider than LV, and a more marked mid-dorsal crest on shoulder's top. Wuchiapingian specimens are, proportionally, slightly longer, DB is also longer. DB of Wuchiapingian 


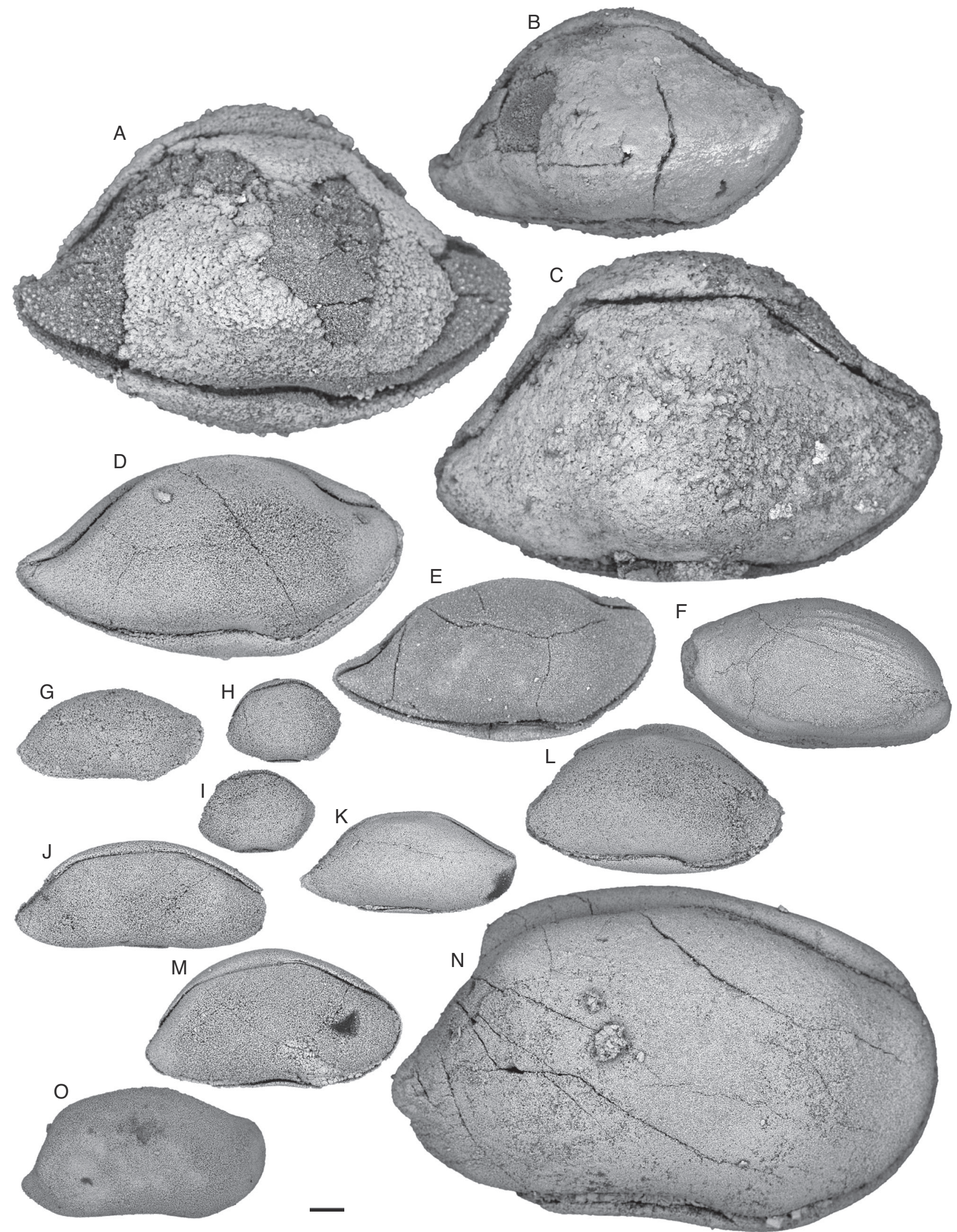

FiG. 6. - Ostracods from Guadalupian-Lopingian boundary interval in South China: A-C, Ceratobairdia? ambigua Ishizaki, 1964; A, P6M3610, 11CHAO88; B, P6M3611, 11CHAO88; C, P6M3612, 11CHAO88; D, E, Bairdia chaotianensis Zazzali, n. sp.; D, holotype, P6M3613, 11CHAO172; E, paratype, P6M3614, 11CHAO88; F, Bairdia sp. 1, P6M3615, sample 11CHAO173; G, Bairdia sp. 2, P6M3616, sample 11CHAO173; H, I, Bairdia sp. 3; H, P6M3617, sample 11CHAO171; I, P6M3618, sample 11CHAO171; J, Bairdia sp. 4, P6M3619, sample 11CHAO172; K, Bairdia sp. 5, P6M3620, sample 11CHAO171; L, Bairdia sp. 6, P6M3621, sample 11CHAO171; M, Bairdia sp. 7, P6M3622, sample 11CHAO171; N, O, Bairdia sp. 8; N, P6M3623, sample 11CHAO170; O, P6M3624, sample 11CHAO165. All photographs represent complete carapaces in right lateral view, except $\mathbf{F}$ in left lateral view. Scale bar: $100 \mu \mathrm{m}$. 
specimens is about the half of $\mathrm{L}$ and about the third of $\mathrm{L}$ for Capitanian ones.

The presence of DB shoulder and crest on each valve distinguishes $B$. chaotianensis Zazzali, n. sp. from all the other close species. Bairdia chaotianensis Zazzali, n. sp. could be compared to B. sp. 9 sensu Cordell (1952) from the Late Pennsylvanian (Late Carboniferous) of Missouri (USA; Cordell 1952) by its general lateral RV shape. It differs here by the absence of overlap at $\mathrm{DB}$, more concave $\mathrm{ADB}$ and $\mathrm{PDB}$ and by more rounded sides in dorsal view. Bairdia chaotianensis Zazzali, n. sp. is also close to Orthobairdia oklahomensis (Harlton, 1927) (in Melnyk \& Maddocks 1988b) from the Late Pennsylvanian (Late Carboniferous) of Texas (USA) by RV general lateral outline. It differs here by a PDB more concave at RV, the absence of DB overlap, and a longer posterior end. B. chaotianensis Zazzali, n. sp. differs here from B. urodeloformis Chen, 1987 (in Crasquin et al. 2010) from latest Permian of Meishan section, Zhejiang province, China, by its $\mathrm{PB}$ and $\mathrm{AB}$ smaller radius of curvature. It differs also here by an arched $\mathrm{DB}$, an $\mathrm{ADB}$ deeply concave in right lateral view and a maximum of overlapping located at VB.

Internal characters could not be observed due to the infilling and preservation.

Genus Ceratobairdia Sohn, 1954

Type SPECIES. - Ceratobairdia dorsospina Sohn, 1954 by original designation.

\section{Ceratobairdia? ambigua Ishizaki, 1964}

(Fig. 6A-C)

Ceratobairdia? ambigua Ishizaki, 1964: 155, pl.19, fig. 6a, b (non figs 7,8$)$.

Bairdia guangxiensis Guan in Guan et al., 1978: 154, pl. 38, figs 3, 4. - Wang 1978: 291 pl. 3, fig. 5. — Chen \& Shi 1982: 122, pl. 5, fig. 17. - Wei et al. 1983: 56, pl. 15, fig. 6. - Chen \& Bao 1986: 114, pl. 3, figs 7, 8. - Shi \& Chen 1987: 31, pl. 3, figs 1-9; 2002: 67, pl. 5, figs 1-9; pl. 28, figs 1, 2.

Bairdia fujisan Tanaka \& Maeda in Tanaka et al., 2012: 96, fig. 6.3.

Bairdia nishiwakii Tanaka \& Nishimura in Tanaka et al., 2013: 297, fig. 8.1 (non fig. 2).

MATERIAL EXAMINED. - Five complete carapaces, ten broken carapaces, five isolated valves and three broken valves.

DimENSIONS. - $\mathrm{L}=1020-1570 \mu \mathrm{m} ; \mathrm{H}=666-974 \mu \mathrm{m}$.

OcCurRences. - Kitakami Massif, North-East Japan, Middle Permian (Ishizaki 1964); Guangxi Province, China, Late Permian (Guan et al. 1978); Guizhou province, China, Late Permian (Wang 1978); Jiangsu Province, China, latest Permian (Chen \& Shi 1982); Guizhou Province, China, Late Permian (Wei et al. 1983); Jiangsu Province, China, Early Permian (Chen \& Bao 1986); Zhejiang Province, China, latest Permian (Shi \& Chen 1987); Guangxi, China, Late Permian (Shi \& Chen 2002); Gifu Prefecture, Japan, Early Permian (Tanaka et al. 2012); Gifu Prefecture, Japan, Middle Permian (Tanaka et al. 2013); Chaotian section, Sichuan Province, China, basal Wuchiapingian, Late Permian (this study; sample: 11CHAO88, see Table 1).
REMARKS

Ceratobairdia? ambigua Ishizaki, 1964 shows a strong dorsal and ventral overlap. L of DB is about the third of carapace $L$. $\mathrm{DB}$ and VB are nearly straight at RV and slightly convex to nearly straight at LV. The maximum thickness is located at mid-L, slightly below midventral part of valves. In anterior and posterior views, VB is quite flat and bounded by a ventral ridge or alae. This structure, added to the flat ventral area, leads to a specific subtriangular shape that could be observed in anterior/posterior view. A sinuous midventral contact margin is also observed. We suggest then that Bairdia guangxiensis Guan, 1978, Bairdia fujisan Tanaka \& Maeda, 2012 and Bairdia nishiwakii Tanaka \& Nishimura, 2013 are junior synonyms of C.? ambigua Ishizaki, 1964.

Suborder SIGILLIOCOPINA Martens, 1992

Superfamily SIGILLOIDEA Mandelstam, 1960

Family Microcheilinellidae Gramm, 1975

Genus Microcheilinella Geis, 1933

TYPE SPECIES. — Microcheilus distortus Geis, 1932 by original designation.

Microcheilinella wujiapingensis Zazzali, n. sp. (Fig. $11 \mathrm{G}, \mathrm{H}$ )

TYPE MATERIAL. - Holotype: one complete carapace (P6M3690; Fig. 11G); paratype: one complete carapace (P6M3691; Fig. 11H).

Etymology. - From the Type horizon, Wujiaping Formation.

MATERIAL EXAMINED. - Four complete carapaces and three broken carapaces.

Dimensions. - LV: L=524-567 $\mu \mathrm{m}$; $\mathrm{H}=353-386 \mu \mathrm{m}$. Holotype: $\mathrm{L}=535 \mu \mathrm{m} ; \mathrm{H}=370 \mu \mathrm{m}$. Paratype: $\mathrm{L}=531 \mu \mathrm{m} ; \mathrm{H}=386 \mu \mathrm{m}$.

TyPe HORIZON. - Sample 11CHAO88, Wujiaping Formation, basal Wuchiapingian, Late Permian.

TyPe LOCALITY. - Chaotian section $\left(30^{\circ} 23.713 \mathrm{~N}, 106^{\circ} 49.615 \mathrm{E}\right)$, Sichuan Province, P. R. China.

Occurrences. - Chaotian section, Sichuan Province, China, Wujiaping Formation, basal Wuchiapingian, Late Permian (sample: 11CHAO88, see Table 1).

Diagnosis. - A species of Microcheilinella with medium sized carapace. Both valves present prominent mid-dorsal shoulder. Maximum W located behind mid-L at RV and in front of mid-L at LV.

\section{DESCRIPTION}

LV overlaps RV all around the carapace: very slightly at DB, narrowly at PDB, PVB and AVB, distinctly at $\mathrm{ADB}, \mathrm{AB}, \mathrm{PB}$ and VB. Overlap maximum at VB.

Hinge line straight, equalling about two third of RV length and about half the LV length, located well below DB of both valves which present prominent mid-dorsal shoulder.

RV: DB nearly straight and mid-dorsal shoulder broadly convex in lateral view. PDB and PB slightly flattened laterally. 


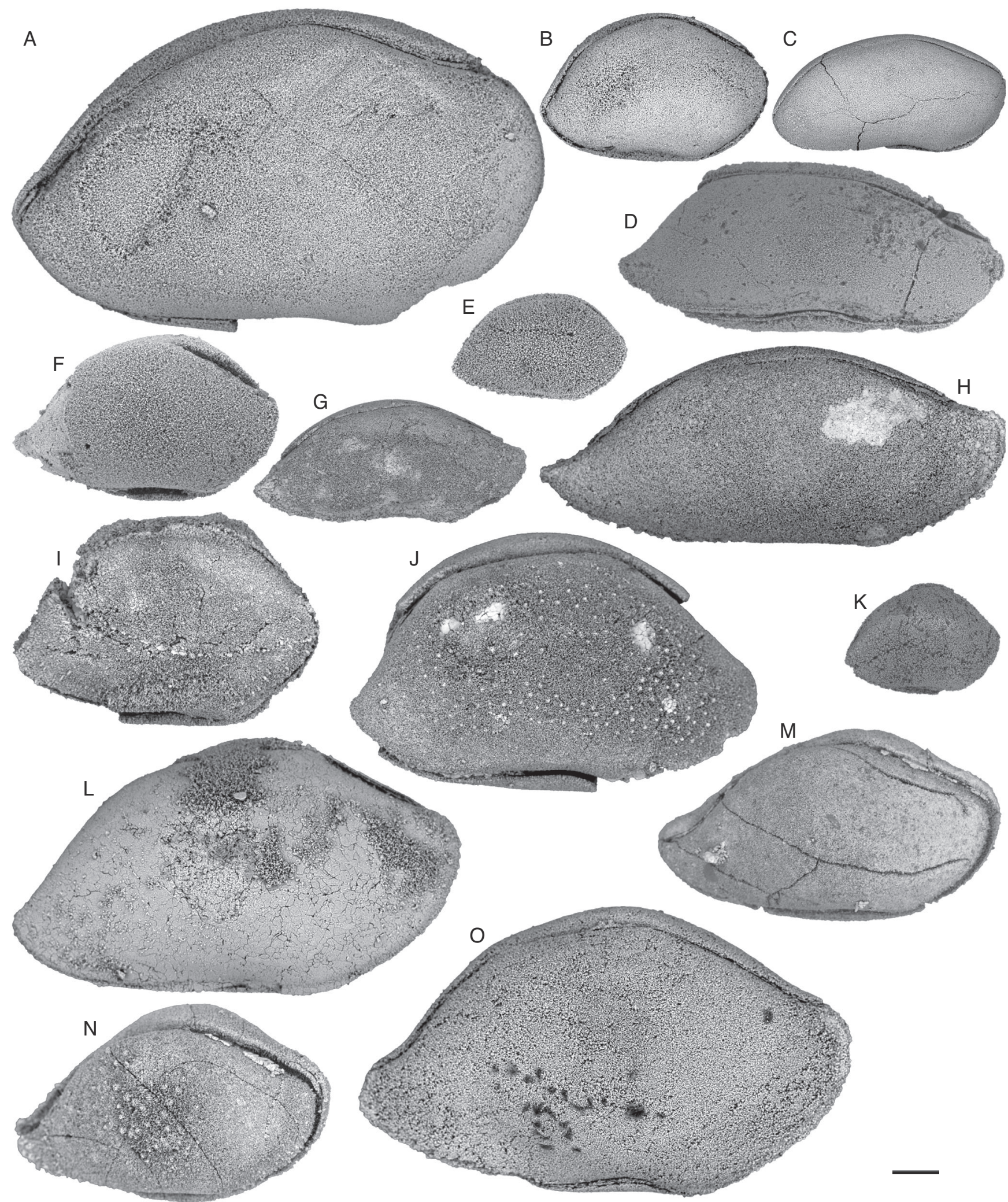

FiG. 7. - Ostracods from Guadalupian-Lopingian boundary interval in South China: A, Bairdia sp. 9, P6M3625, sample 11CHAO168; B, Bairdia sp. 10, P6M3626, sample 11CHAO165; C, Bairdia sp. 11, P6M3627, sample 11CHAO166; D, Bairdia sp. 12, P6M3628, sample 11CHAO163; E, Bairdia sp. 13, P6M3629, sample 11CHAO81; F, Bairdia sp. 14, P6M3630, sample 11CHAO82; G, H, Bairdia sp. 15; G, P6M3631, sample 11CHAO95; H, P6M3632, sample 11CHAO99; I, Bairdia sp. 16, P6M3633, sample 11CHAO85; J, Bairdia sp. 17, P6M3634, sample 11CHAO82; K, Bairdia sp. 18, P6M3635, sample 11CHAO83; L, Bairdia sp. 19, P6M3636, sample 11CHAO87; M, N, Bairdia sp. 20; M, P6M3637, sample 11CHAO88; N, P6M3638, sample 11CHAO88; O, Bairdia sp. 21, P6M3639, sample 11CHAO95. All photographs represent complete carapaces in right lateral view. Scale bar: $100 \mu \mathrm{m}$. 

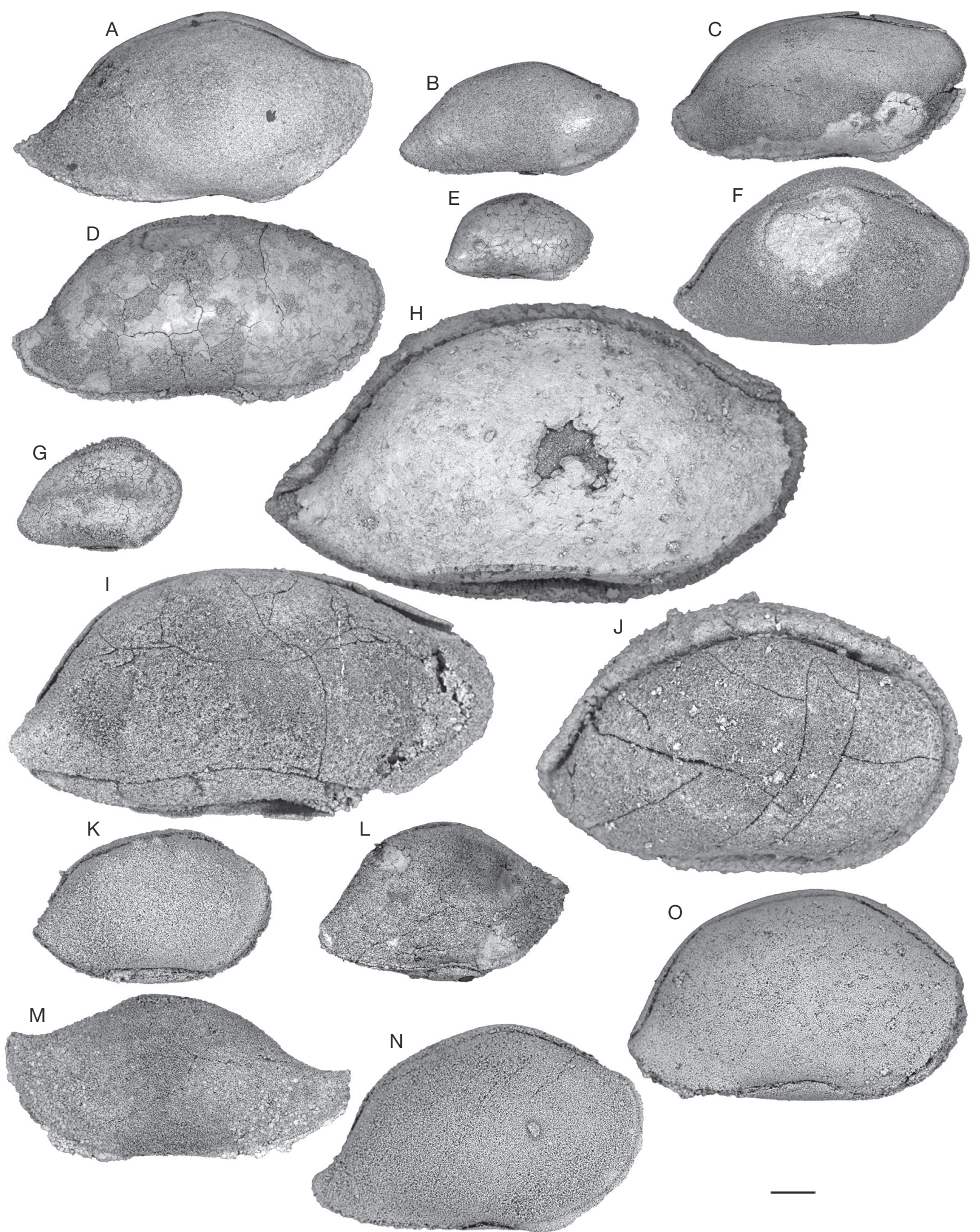

FIG. 8. - Ostracods from Lopingian in South China: A, Bairdia sp. 22, P6M3640, sample 11CHAO96; B, Bairdia sp. 23, P6M3641, sample 11CHAO99; C, D, Bairdia sp. 24; C, P6M3642, sample 11CHA087; D, P6M3643, sample 11CHAO100; E, Bairdia sp. 25, P6M3644, sample 11CHAO95; F, Bairdia sp. 26, P6M3645, sample 11CHAO100; G, Bairdia sp. 27, P6M3646, sample 11CHAO100; H, Bairdia sp. 28, P6M3647, sample 11CHAO88; I, Bairdia sp. 29, P6M3648, sample 11CHAO88; J, K, Bairdia sp. 30; J, P6M3649, sample 11CHAO88; K, P6M3650, sample 11CHAO95; L, Bairdia sp.31, P6M3651, sample 11CHAO100; M, Bairdia sp. 32, P6M3652, sample 11CHAO92; N, Bairdia sp. 33, P6M3653, sample 11CHAO95; O, Bairdia sp. 34, P6M3654, sample 11CHAO95. All photographs represent complete carapaces in right lateral view, except $\mathbf{M}$ which is a left valve in left lateral view. Scale bar: $100 \mu \mathrm{m}$. 


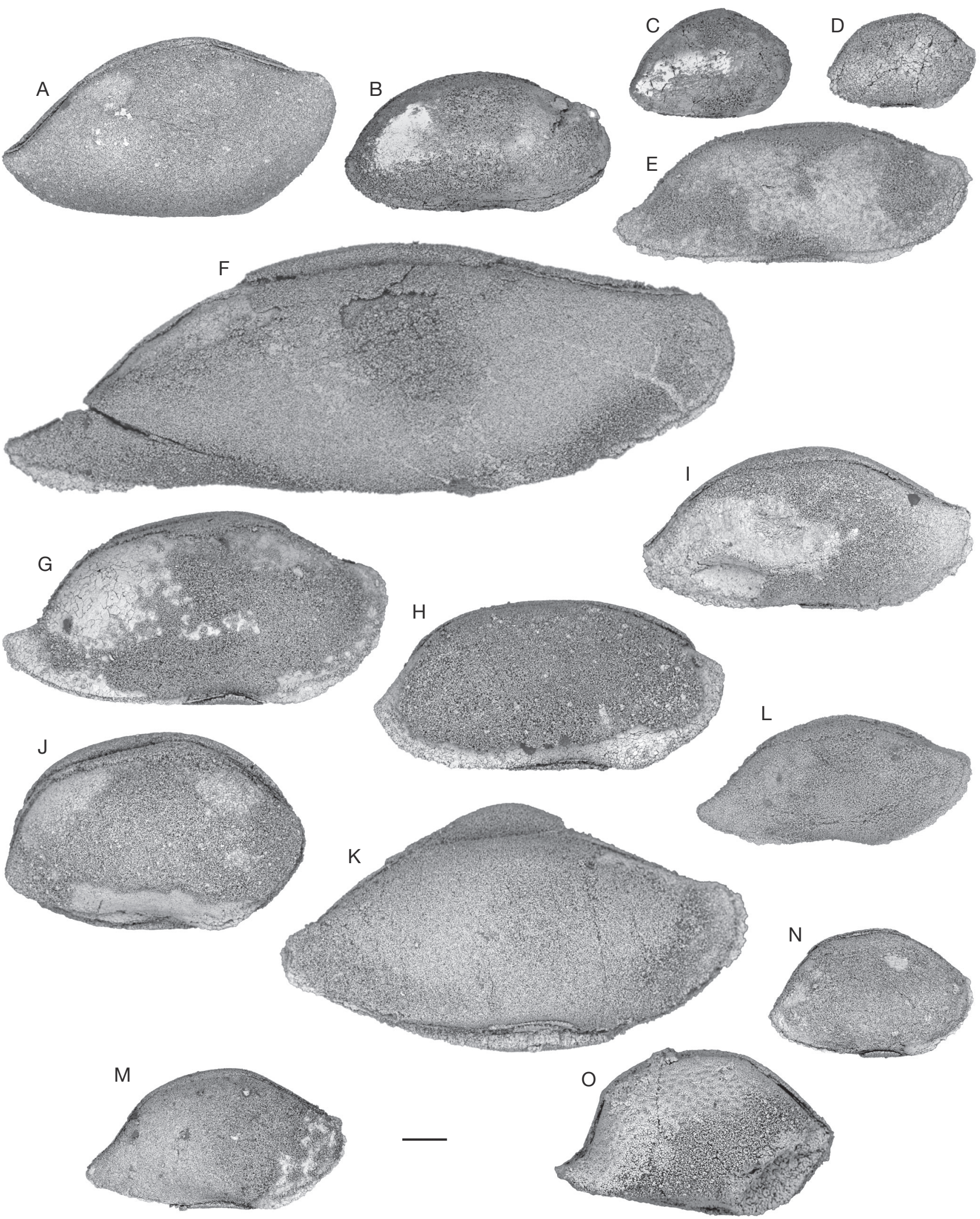

FIG. 9. - Ostracods from Lopingian in South China: A, Bairdia sp. 35, P6M3655, sample 11CHAO99; B, Bairdia sp. 36, P6M3656, sample 11CHAO99; C, Bairdia sp. 37, P6M3657, sample 11CHAO97; D, Bairdia sp. 38, P6M3658, sample 11CHAO100; E, Bairdia sp.39, P6M3659, sample 11CHAO99; F, Bairdia sp. 40, P6M3660, sample 11CHAO99; G, Bairdia sp. 41, P6M3661, sample 11CHAO99; H, Bairdia sp. 42, P6M3662, sample 11CHAO99; I, Bairdia sp. 43, P6M3663, sample 11CHAO99; J, Bairdia sp. 44, P6M3664, sample 11CHAO99; K, Bairdia sp. 45, P6M3665, sample 11CHAO100; L, Bairdia sp. 46, P6M3666, sample 11CHAO100; M, Bairdia sp. 47, P6M3667, sample 11CHAO100; N, Bairdia sp. 48, P6M3668, sample 11CHAO100; O, Cetollina sp. 1, PM3775, sample 11CHAO95. All photographs represent complete carapaces, except $\mathbf{O}$ which is a broken one, all in right lateral view. Scale bar: $100 \mu \mathrm{m}$. 


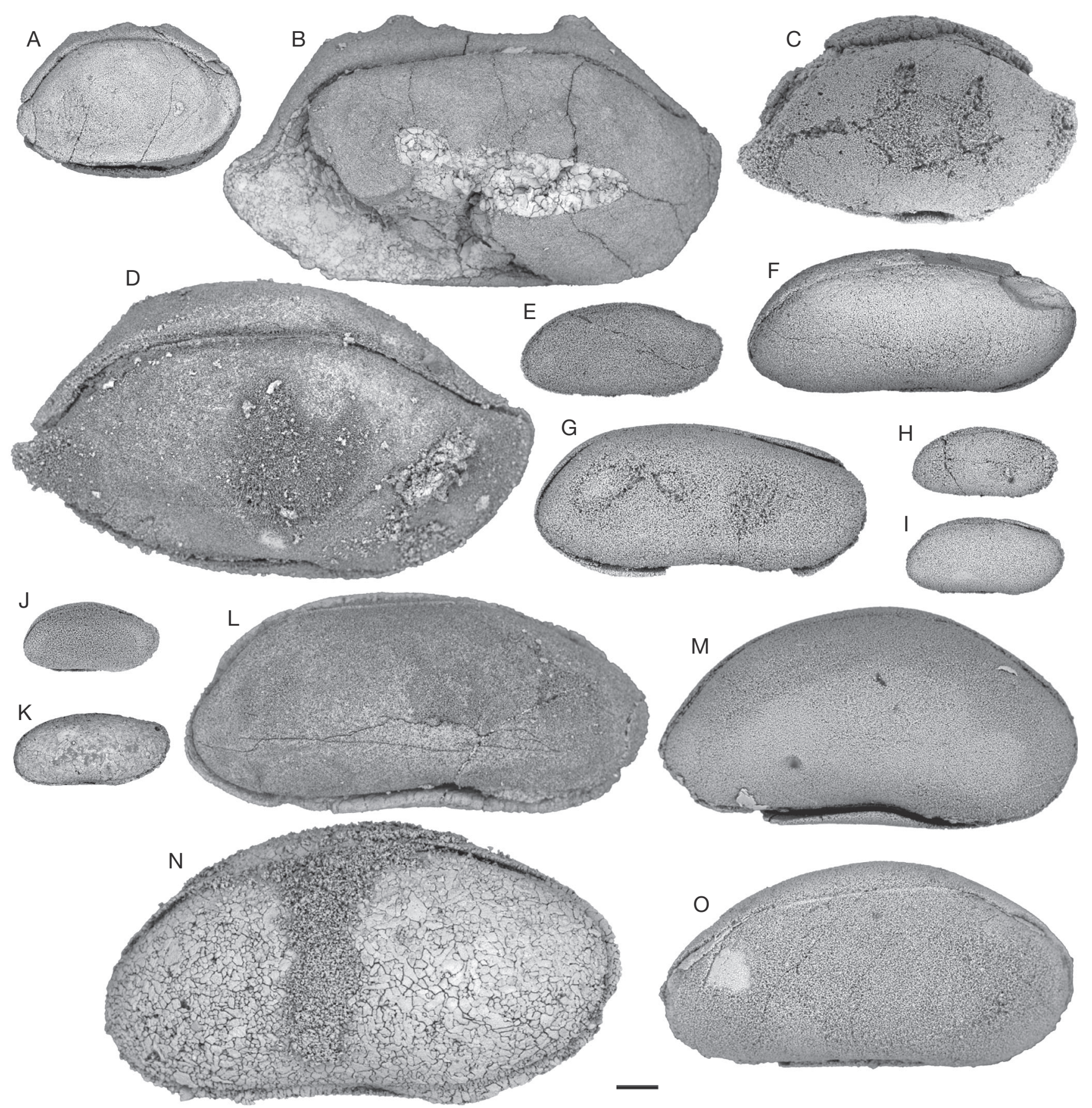

FIG. 10. - Ostracods from Guadalupian-Lopingian boundary interval in South China: A, Mirabairdia sp. 1, P6M3669, sample 11CHAO173; B, Mirabairdia sp. 2, P6M3670, sample 11CHA097; C, Petasobairdia sp. 1, P6M3671, sample 11CHAO82; D, Petasobairdia sp. 2, P6M3672, sample 11CHAO88; E, Bairdiacypris sp. 1, P6M3673, sample 11CHAO171; F, Bairdiacypris sp. 2, P6M3674, sample 11CHAO173; G, Bairdiacypris sp. 3, P6M3675, sample 11CHAO172; H, Bairdiacypris? sp. 4, P6M3676, sample 11CHAO169; I, Bairdiacypris sp. 5, P6M3677, sample 11CHAO166; J, Bairdiacypris sp. 6, P6M3678, sample 11CHAO80; K, Bairdiacypris sp. 7, P6M3679, sample 11CHA087; L, Bairdiacypris sp. 8, P6M3680, sample 11CHAO88; M, Bairdiacypris? sp. 9, P6M3681, sample 11CHAO95; N, Bairdiacypris sp. 10, P6M3682, sample 11CHAO99; O, Bairdiacypris sp. 11, P6M3683, sample 11CHAO100. All photographs represent complete carapaces in right lateral view. Scale bar: $100 \mu \mathrm{m}$.

PB with small radius of curvature. PB maximum convexity located at mid-H. Ventral margin broadly convex. AB broadly rounded with maximum convexity at mid-H. Greatest L located at mid-H. Greatest $\mathrm{H}$ located slightly anterior to mid-L.

LV: DB nearly straight. Mid-dorsal shoulder more prominent than at RV. Anterior part of the shoulder nearly straight. $\mathrm{ADB}$ and $\mathrm{AB}$ broadly rounded with maximum convexity located slightly below mid-H. Posterior part of the shoulder slightly concave at median part. PDB nearly straight. PB with small radius of curvature, smaller than RV one. $\mathrm{PB}$ maximum convexity located slightly above mid-H. Ventral margin more convex at LV than at RV. Greatest L located slightly above mid-H at LV. Greatest $\mathrm{H}$ located slightly anterior to mid-L. 


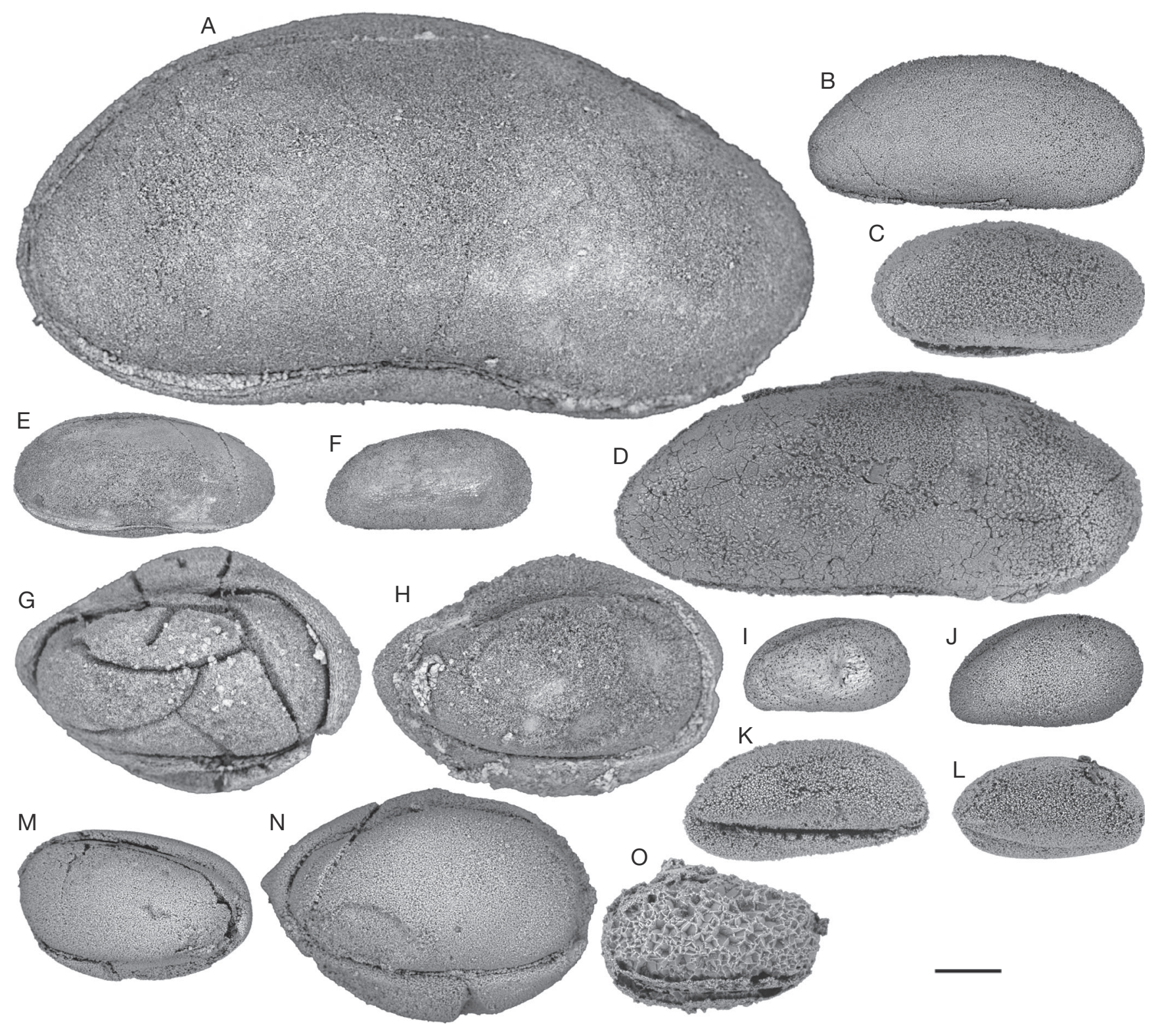

FIG. 11. - Ostracods from Guadalupian-Lopingian boundary interval in South China: A, Bairdiacypris? sp. 12, P6M3684, sample 11CHAO95; B, Fabalicypris sp. 1, P6M3685, sample 11CHAO172; C, Fabalicypris sp. 2, P6M3686, sample 11CHAO82; D, Fabalicypris sp. 3, P6M3687, sample 11CHAO83; E, Fabalicypris sp. 4, P6M3688, sample 11CHAO95; F, Fabalicypris sp. 5, P6M3689, sample 11CHAO95; G, H, Microcheilinella wujiapingensis Zazzali, n. sp.; G, holotype, P6M3690, 11CHAO88; H, paratype, P6M3691, 11CHAO88; I, J, Microcheilinella pagodaensis Zazzali, n. sp.; I, holotype, P6M3692, 11CHAO99; J, paratype, P6M3693, 11CHAO166; K, L, Microcheilinella rectodorsata? Forel, 2010; K, P6M3694, 11CHAO81; L, P6M3695, 11CHAO80; M, Microcheilinella sp. 1, P6M3696, sample 11CHAO172; N, Microcheilinella sp. 2, P6M3697, sample 11CHAO167; O, Microcheilinella? sp. 3, P6M3698, sample 11CHAO156. All photographs represent complete carapaces, except $\mathbf{O}$ which is a broken one, all in right lateral view. Scale bar: $100 \mu \mathrm{m}$.

Carapace asymmetric in dorsal view with W max located behind mid-L at RV and posterior to mid-L at LV. RV thicker than LV.

\section{REMARKS}

Microcheilinella wujiapingensis Zazzali, n. sp. is close to M. venusta Chen, 1958 from the Early Permian of South China (Chen 1958). It differs here by its DB which is nearly straight unlike the convex one of $M$. venusta Chen, 1958, and by its mid-dorsal shoulder present on both valves. VB, at RV, is here broadly convex but seems nearly straight for $M$. venusta. $\mathrm{VB}$, at $\mathrm{LV}$, is here more convex than $M$. venusta one. Microcheilinella venusta maximum $\mathrm{W}$ is located near mid-L at both valves, whereas $M$. wujiapingensis Zazzali, n. sp. maximum $\mathrm{W}$ is located behind mid-L at RV and posterior to mid-L at LV. RV thicker than LV.

Microcheilinella wujiapingensis Zazzali, n. sp. is close to M. venusta Chen, 1958 (sensu Chen \& Shi 1982. In this paper, the species is different from the original one) from latest Permian of South China, by its LV and RV general outline in lateral view, its mid-dorsal shoulder on each valves, the LV ends radius of curvature and maximum on convexity position and the general overlap. It differs by the maximum W, located in the anterior quarter of both valves for $M$. venusta (sensu Chen \& Shi 1982) in dorsal view. 
Microcheilinella pagodaensis Zazzali, n. sp. (Figs 11I, J; 19)

TYPE MATERIAL. - Holotype: one complete carapace (P6M3692; Fig. 11I); paratype: one complete carapace (P6M3693; Fig. 11J).

ETYMOLOGY. - Reference to the pagoda at the top of the Chaotian section.

MATERIAL EXAMINED. - 22 complete carapaces and four broken carapaces.

Dimensions. $-\mathrm{L}=209-350 \mu \mathrm{m} ; \mathrm{H}=110-187 \mu \mathrm{m}$. Holotype: $\mathrm{L}=263 \mu \mathrm{m} ; \mathrm{H}=138 \mu \mathrm{m}$. Paratype: $\mathrm{L}=306 \mu \mathrm{m} ; \mathrm{H}=169 \mu \mathrm{m}$ (Fig. 19).

TyPE HORIZON. - Sample 11CHAO99, Wujiaping Formation, basal Wuchiapingian, Late Permian.

TyPe LOCALITY. - Chaotian section $\left(30^{\circ} 23.713 \mathrm{~N}-106^{\circ} 49.615 \mathrm{E}\right)$, Sichuan Province, P. R. China.

OCCuRrenCES. - Chaotian section, Sichuan Province, China, Maokou and Wujiaping Formations, Middle Capitanian-basal Wuchiapingian, Middle-Late Permian (samples: 11CHAO 173, 11CHAO172, 11CHAO171, 11CHAO166, 11CHAO84, 11CHAO90, 11CHAO92, 11CHAO97, 11CHAO99, 11CHAO100, see Table 1).

DiAGNOSIS. - A species of Microcheilinella with small sized carapace. $\mathrm{RV}$ anterior half significantly higher than posterior one in lateral view. Maximum W located behind mid-L at RV and in front of mid-L at LV.

\section{DESCRIPTION}

LV overlaps RV all around the carapace: very slightly at AVB and $\mathrm{VB}$, narrowly at $\mathrm{AB}, \mathrm{ADB}, \mathrm{PDB}$ and $\mathrm{PB}$, distinctly at $\mathrm{DB}$.

Hinge line straight, equalling about half $\mathrm{L}$.

In right lateral view: $\mathrm{DB}$ slightly arched to nearly straight at RV and broadly convex at LV. PDB arched. PB with small radius of curvature. $\mathrm{PB}$ maximum convexity located below mid-H. PVB convex. VB slightly concave to nearly straight. AVB broadly convex. AB broadly rounded with maximum convexity located at mid-H. Greatest L located near mid$\mathrm{H}$. Greatest $\mathrm{H}$ anterior to mid- $\mathrm{L}$ at $\mathrm{RV}$ and near mid- $\mathrm{L}$ at LV. RV anterior half significantly higher than posterior one.

In dorsal view: posterior extremities rounded, anterior extremities slender. Maximum W located behind mid-L at $\mathrm{RV}$ and in front of mid-L at LV.

\section{REMARKS}

The new species is quite different from all the previously described species. The closest species is an unpublished species, $M$. sp. 10 (Chitnarin 2010, unpublished $\mathrm{PhD}$ thesis; pl. 16, fig. 7), from the Middle Permian of Thailand. This last species has the same general outline. Here PB radius of curvature at $\mathrm{RV}$ is smaller, the overlap less marked at $\mathrm{PDB}$ and $\mathrm{ADB}$ and the RV anterior half significantly higher than posterior one.

\section{Microcheilinella rectodorsata? Forel, 2010}

(Fig. $11 \mathrm{~K}, \mathrm{~L}$ )

Microcheilinella rectodorsata Forel in Crasquin et al., 2010: 359, fig. 22A-G.
Material EXAMINED. - Six complete carapaces and two broken carapaces.

Dimensions. $-\mathrm{L}=292-416 \mu \mathrm{m} ; \mathrm{H}=163-235 \mu \mathrm{m}$.

Occurrences. - Meishan section, Changxing Formation, Meishan Member, Changhsingian, Late Permian (Crasquin et al. 2010); ?Chaotian section, Sichuan Province, China, Wujiaping Formation, basal Wuchiapingian, Late Permian (this study, samples: 11CHAO80, 11CHAO81, 11CHAO85, 11CHAO95, 11CHAO99, 11CHAO100, see Table 1).

\section{REMARKS}

Our specimens are very similar to $M$. rectodorsata Forel, 2010 from the Late Permian of China (Crasquin et al. 2010) but differ in having longer carapace, less marked overlapping and more acute $\mathrm{PB}$.

Order PALAEOCOPIDA Henningsmoen, 1953

Suborder BEYRICHICOPINA Scott, 1961

Superfamily APARCHITOIDEA Jones, 1901

Family APARCHITIDAE Jones, 1901

Genus Cyathus Roth \& Skinner, 1930

TYPe SPECIES. - Cyathus ulrichi Roth \& Skinner, 1930 by original designation.

\section{Cyathus caperata? (Guan, 1978)}

(Fig. 14E, F)

Sinocoelonella caperata Guan in Guan et al., 1978: 149, pl. 37, fig. 17, pl. 38, fig. 1 .

Cyathus caperata - Chen \& Bao 1986: 111, pl. 4, fig. 3. - Shi \& Chen 1987: 32, pl. 10, figs 10-18. - Yuan et al. 2009:388, 392, pl. 1, fig. 15. - Crasquin et al. 2010: 332-334, fig. 3A-D. - Chitnarin et al. 2012: 806, fig. 4A, B, D, E. - Burrett et al. 2014: 15-16, fig. 12s, t.

MATERIAL EXAMINED. - Eight complete carapaces and two broken carapaces.

Dimensions. $-\mathrm{L}=276-453 \mu \mathrm{m} ; \mathrm{H}=174-255 \mu \mathrm{m}$.

OCCURRENCES. - Wugang, Hunan Province, China, Early Permian (Guan et al. 1978); Chisia Formation, Jiangsu Province, China, Early Permian (Chen \& Bao 1986); Meishan section, Baoqing and Meishan Members, Changxing Formation, Zhejiang Province, China, Late Permian (Shi \& Chen 1987; Crasquin et al. 2010); Saiwa section, Guizhou Province, China, latest Permian (Yuan et al. 2009); Chaiyaphum Province, North-eastern Thailand, Early Permian, Phetchabun Province, central Thailand, Early Permian and Nakhon Sawan Province, central Thailand, Middle Permian (Chitnarin et al. 2012); E-Lert Formation, Thailand, Early Permian (Burrett et al. 2014); ?Chaotian section, Sichuan Province, China, Maokou Formation, Middle Capitanian, Middle Permian (this study, samples: $11 \mathrm{CHAO}$ 173, 11CHAO171, 11CHAO170, 11CHAO167, see Table 1).

\section{REMARKS}

Our specimens are very similar to C. caperata (Guan, 1978) from the Permian of Thailand and China (see above) but differ in having longer and less arched carapaces in dorsal view and less prominent DB inflation. 


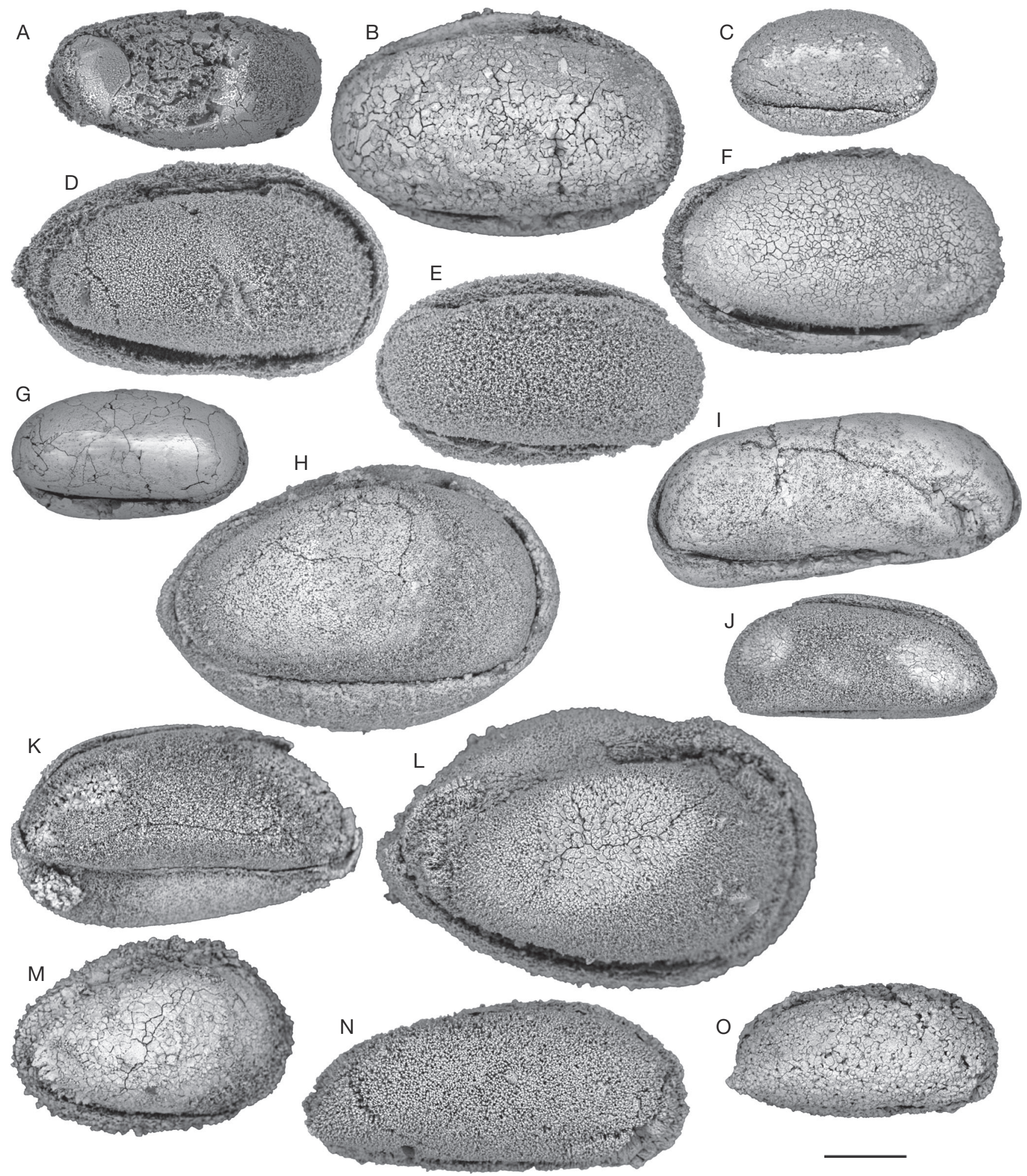

FIG. 12. - Ostracods from Guadalupian-Lopingian boundary interval in South China: A, Microcheilinella? sp. 4, P6M3699, sample 11CHAO158; B, Microcheilinella sp. 5, P6M3700, sample 11CHAO97; C, Microcheilinella sp. 6, P6M3701, sample 11CHAO97; D, Microcheilinella sp. 7, P6M3702, sample 11CHAO81; E, Microcheilinella sp. 8, P6M3703, sample 11CHAO81; F, Microcheilinella sp. 9, P6M3704, sample 11CHAO87; G, Microcheilinella sp. 10, P6M3705, sample 11CHAO87; H, Microcheilinella sp. 11, P6M3706, sample 11CHAO95; I, Microcheilinella sp. 12, P6M3707, sample 11CHAO87; J, K, Microcheilinella sp. 13; J, P6M3708, sample 11CHAO95; K, P6M3709, sample 11CHAO88; L, Microcheilinella sp. 14, P6M3710, sample 11CHAO100; M, Microcheilinella sp. 15, P6M3711, sample 11CHAO100; N, Microcheilinella sp. 16, P6M3712, sample 11CHAO90; O, Microcheilinella sp. 17, P6M3713, sample 11CHAO100. All photographs represent complete carapaces in right lateral view, except $\mathbf{A}$ and $\mathbf{L}$ which are broken. Scale bar: $100 \mu \mathrm{m}$. 


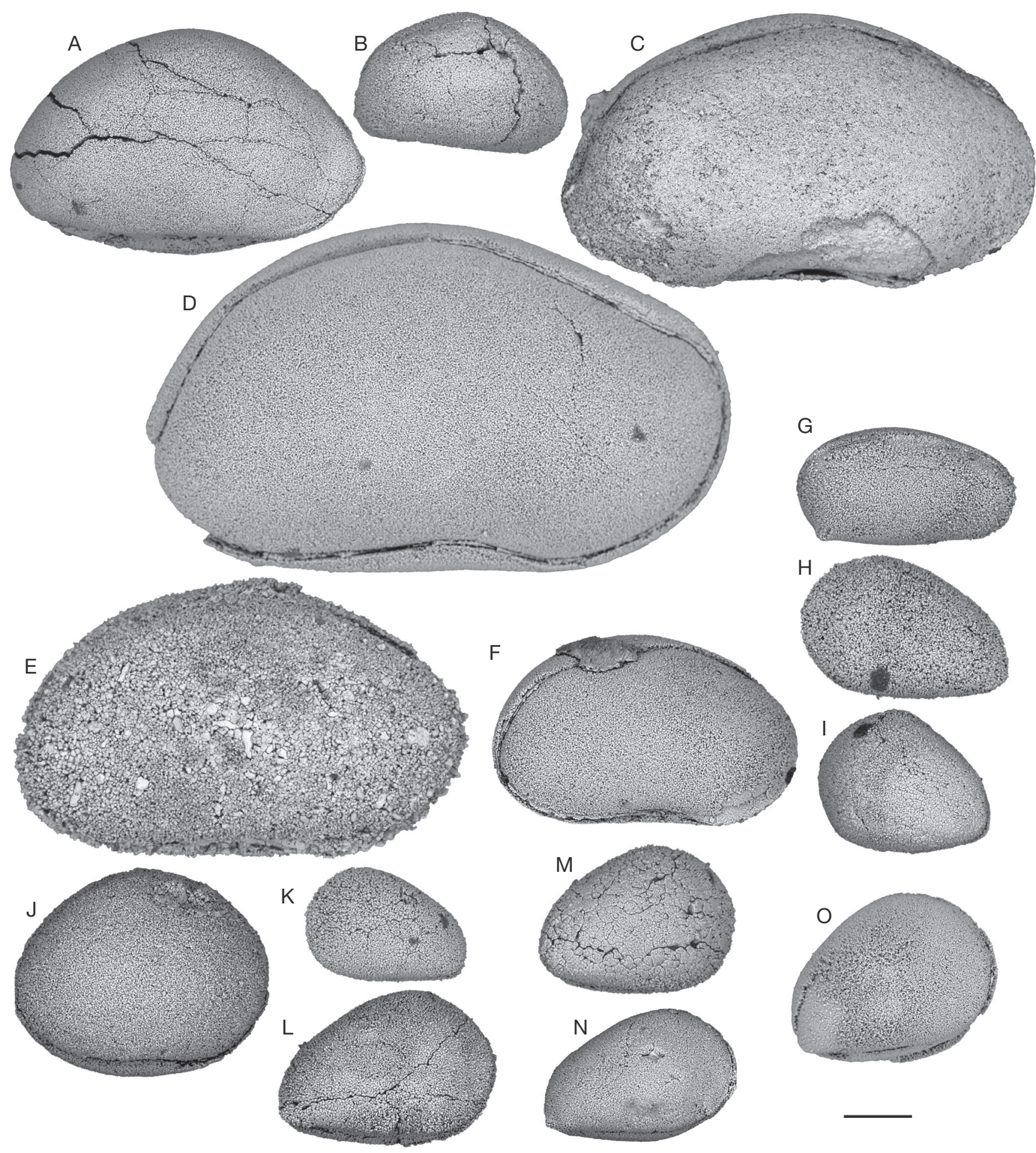

FIG. 13. - Ostracods from Guadalupian-Lopingian boundary interval in South China: A, Silenites? sp. 1, right lateral view, P6M3714, sample 11CHAO173; B, Silenites? sp. 2, right lateral view, P6M3715, sample 11CHAO172; C, Silenites? sp. 3, right lateral view, P6M3716, sample 11CHAO97; D, Silenites? sp. 4, right lateral view, P6M3717, sample 11CHAO87; E, Silenites? sp. 5, right lateral view, P6M3718, sample 11CHAO91; F, Silenites? sp. 6, right lateral view, P6M3719, sample 11CHAO95; G, Waylandella sp. 1, right lateral view, P6M3720, sample 11CHAO165; H, Basslerella sp. 1, left lateral view, P6M3721, sample 11CHAO173; I, Basslerella sp. 2, left lateral view, P6M3722, sample 11CHAO173; J, Basslerella sp. 3, right lateral view, P6M3723, sample 11CHAO173; K, Basslerella? sp. 4, left lateral view, P6M3724, sample 11CHAO171; L, Basslerella sp. 5, right lateral view, P6M3725, sample 11CHAO171; M, Basslerella sp. 6, right lateral view, P6M3726, sample 11CHAO171; N, Basslerella sp. 7, right lateral view, P6M3727, sample 11CHAO168; O, Basslerella sp. 8, right lateral view, P6M3728, sample $11 \mathrm{CHAO87}$. All photographs represent complete carapaces. Scale bar: $100 \mu \mathrm{m}$. 

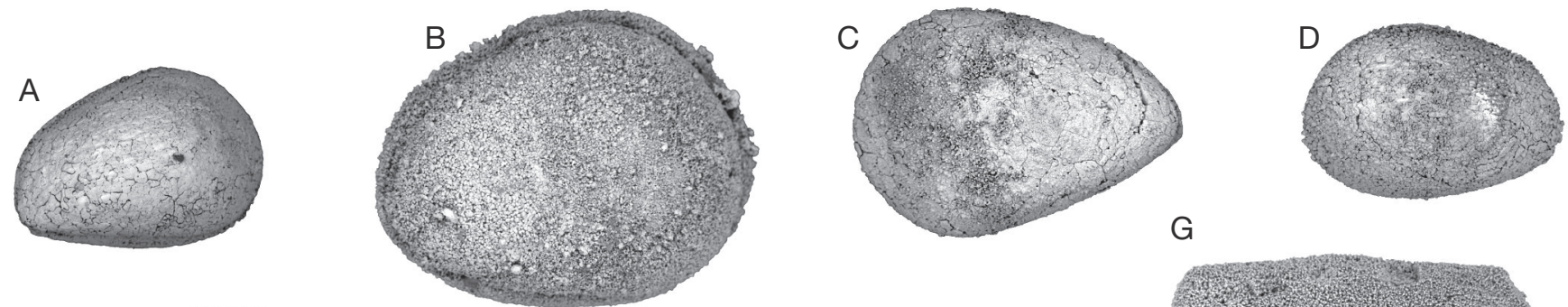

G
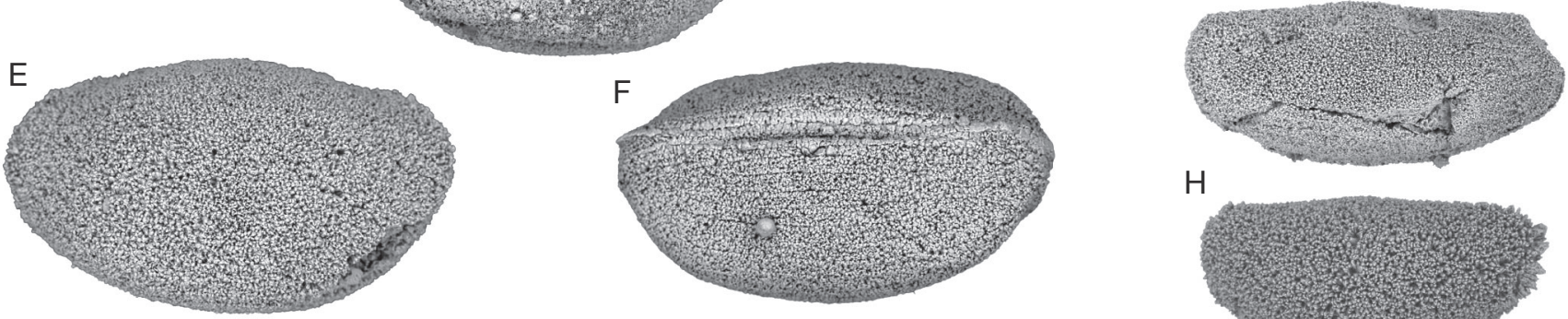

$\mathrm{H}$

1
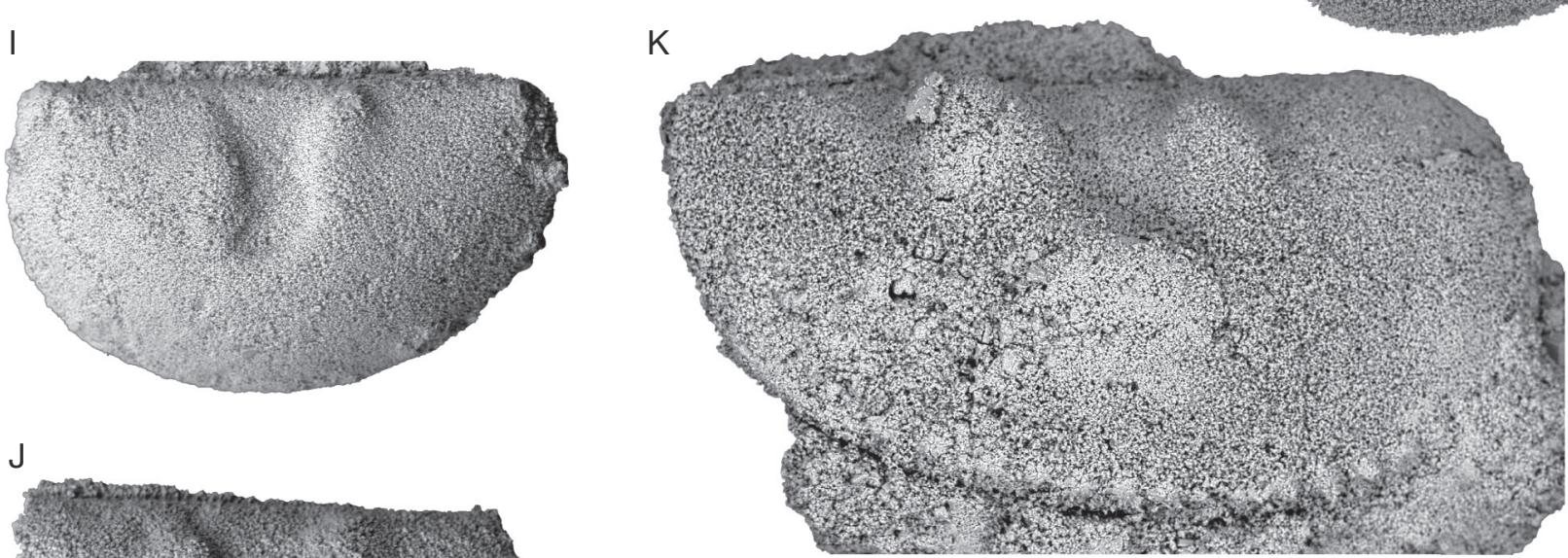

ง
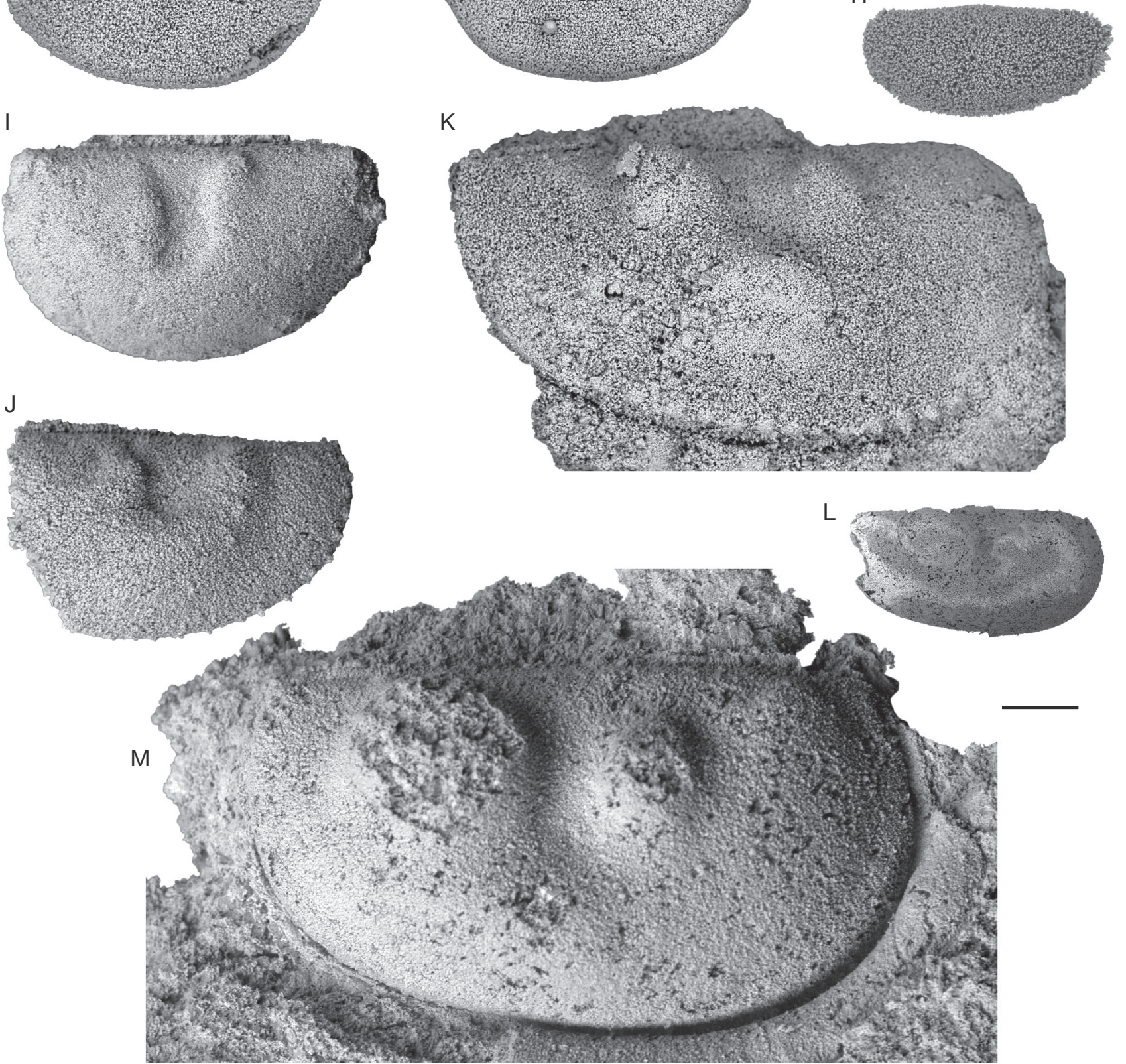

FIG. 14. - Ostracods from Guadalupian-Lopingian boundary interval in South China: A, Basslerella sp. 9, right lateral view of complete carapace, P6M3729, sample 11CHAO 99; B, Basslerella sp. 10, right lateral view of complete carapace, P6M3730, sample 11CHAO85; C, Basslerella sp. 11, left lateral view of complete carapace, P6M3731, sample 11CHAO100; D, Basslerella sp. 12, left lateral view of complete carapace, P6M3732, sample 11CHAO99; E, F, Cyathus caperata? (Guan, 1978); E, right lateral view of complete carapace, P6M3733, 11CHAO173; F, dorsal view of complete carapace, P6M3734, 11CHAO171; G, H, Cyathus elliptica? Shi, 1987; G, left lateral view of complete carapace, P6M3735, 11CHAO79; H, left lateral view of complete carapace, P6M3736, 11CHAO81; I-K, Hollinella martensiformis Crasquin, 2010; I, left lateral view of left valve, P6M3737, 11CHAO166; J, left lateral view of broken left valve, P6M3738, 11CHAO173; K, right lateral view of right valve, P6M3739, sample 11CHAO167; L, M, Hollinella sp. 1; L, right lateral view of broken right valve, P6M3740, sample 11CHAO72; M, right lateral view of broken right valve, P6M3741, sample 11CHAO72. Scale bar: $100 \mu \mathrm{m}$. 
Cyathus elliptica? Shi, 1987

(Fig. 14G, H)

Cyathus elliptica Shi in Shi \& Chen, 1987: 32, pl. 10, figs 20-23; pl. 17, figs 5, 6. - Crasquin et al. 2010: 334, fig. 3E-H. - Chitnarin et al. 2012: 810, fig. 4C, F, G, J. — Burrett et al. 2014: 16, fig. 12u-w.

MATERIAL EXAMINED. - One complete carapace and one broken carapace.

Dimensions. $-\mathrm{L}=334-374 \mu \mathrm{m} ; \mathrm{H}=152-162 \mu \mathrm{m}$.

OCCURRENCES. - Meishan section, Baoqing and Meishan Members, Changxing Formation, Zhejiang Province, China, Late Permian (Shi \& Chen 1987; Crasquin et al. 2010); Chaiyaphum Province, Northeastern Thailand, Early Permian, Phetchabun Province, central Thailand, Early Permian and Nakhon Sawan Province, central Thailand, Middle Permian (Chitnarin et al. 2012); E-Lert Formation, Thailand, Early Permian (Burrett et al. 2014); ?Chaotian section, Sichuan Province, China, Wujiaping Formation, basal Wuchiapingian, Late Permian (this study, samples: 11CHAO79, 11CHAO81, see Table 1).

\section{REMARKS}

Our specimens are attributed to C. elliptica Shi, 1987 from the Permian of China and Thailand (see above) with doubt because of the poor preservation state and the small number of specimens.

Suborder KLOEDENELLOCOPINA Scott, 1961

Superfamily HollinOIDEA Swartz, 1936

Family HOLLINELLidAe Bless \& Jordan, 1971

Genus Hollinella Coryell, 1928

Type SPECIES. - Hollinella dentata Coryell, 1928 by original designation.

Hollinella martensiformis Crasquin, 2010 (Fig. 14I-K)

Hollinella martensiformis Crasquin in Crasquin et al., 2010: 336, fig. 4D-F. — Chitnarin et al. 2012: 828, fig. 19A-D.

MATERIAL EXAMINED. - Two isolated valves and one broken valve.

Dimensions. $-\mathrm{L}=477-521 \mu \mathrm{m} ; \mathrm{H}=286-298 \mu \mathrm{m}$.

OCCURRENCES. - Meishan section, Baoqing and Meishan Member, Changxing Formation, Zhejiang Province, China, Late Permian (Crasquin et al. 2010); Phetchabun Province, Central Thailand, Early Permian and Nakhon Sawan Province, Central Thailand, late Early Permian and Middle Permian (Chitnarin et al. 2012); Chaotian section, Sichuan Province, China, Maokou Formation, Middle Capitanian, Middle Permian (this study, samples: 11CHAO 173, 11CHAO166, see Table 1).

Superfamily KiRKBYOIDEA Ulrich \& Bassler, 1906

Family KIRKBYIDAE Ulrich \& Bassler, 1906

Genus Reviya Sohn, 1961

Type species. - Amphissites? obesus Croneis \& Gale, 1939 by original designation.
Reviya cf. subsompongensis Chitnarin, 2008

(Fig. 16F, G)

Reviya subsompongensis Chitnarin, 2008: 347, fig. 3.14-16.

MATERIAL EXAMINED. - Three isolated valves and one broken valve.

Dimensions. $-\mathrm{L}=469-617 \mu \mathrm{m} ; \mathrm{H}=275-381 \mu \mathrm{m}$.

OcCurrences. - Chaotian section, Sichuan Province, China, Maokou Formation, Middle Capitanian, Middle Permian (this study, samples: 11CHAO 167, 11CHAO166, 11CHAO151, see Table 1).

\section{REMARKS}

General appearance of valves suggests $R$. subsompongensis Chitnarin, 2008 from the Middle Permian of Thailand (Chitnarin et al. 2008, 2012). However here, cardinal angles are more pronounced, $\mathrm{PDB}$ more convex, anterior and posterior flattened extremities more noticeably developed and ornamentation is also slightly different. Perhaps the present species could belong to a geographic subspecies of $R$. subsompongensis.

Superfamily KLOEDENELLOIDEA Ulrich \& Bassler, 1908 Family KNOXITIDAE Egorov, 1950

Genus Geffenina Coryell \& Sohn, 1938

TYPE SPECIES. - Geffenina marmerae Coryell \& Sohn, 1938 by original designation.

\section{Geffenina posterodorsospina? Chitnarin, 2012}

(Fig. 15A, B)

Geffenina posterodorsospina Chitnarin in Chitnarin et al., 2012: 814, fig. 8A-C.

MATERIAl EXAMineD. - Four complete carapaces and three broken carapaces.

Dimensions. $-\mathrm{L}=274-525 \mu \mathrm{m} ; \mathrm{H}=130-305 \mu \mathrm{m}$.

OCCURRENCES. - Phetchabun Province, Early Permian (Chitnarin et al. 2012); ?Chaotian section, Sichuan Province, China, Wujiaping Formation, basal Wuchiapingian, Late Permian (this study, sample: 11CHAO80, see Table 1).

\section{REMARKS}

Our specimens are attributed to G. posterodorsospina Chitnarin, 2012 from the Early Permian of Thailand (Chitnarin et al. 2012) but differ in having less pronounced or absent posterior cardinal angle. Some specimens present a thickened ventromedian area which could be an expression of sexual dimorphism.

\section{Superfamily Paraparchitoidea Scott, 1959}

Family Paraparchitidae Scott, 1959

Genus Samarella Polenova, 1952

TYPE SPECIES. — Samarella crassa Polenova, 1952 by original designation. 

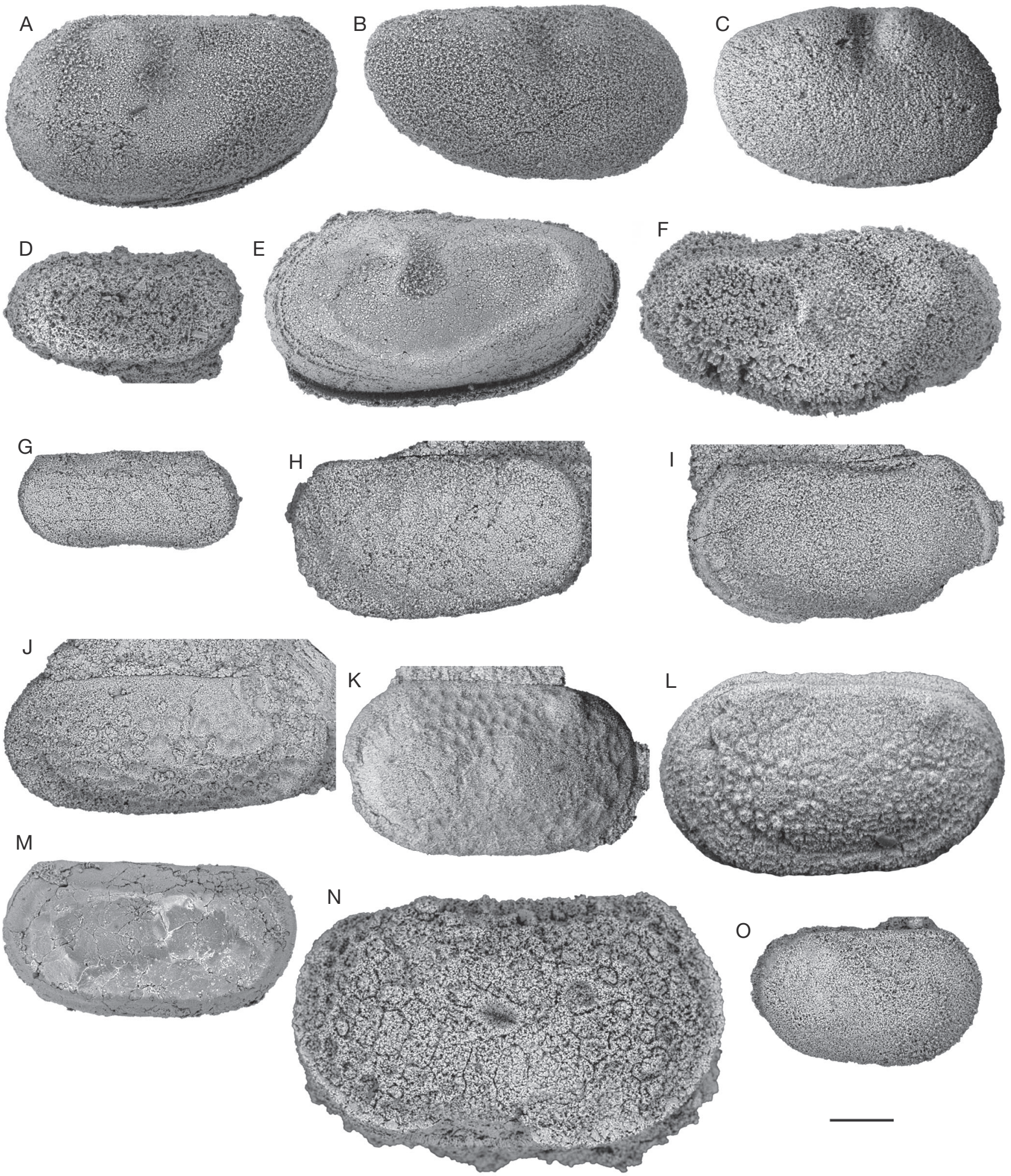

FIG. 15. - Ostracods from Guadalupian in South China: A, B, Geffenina posterodorsospina? Chitnarin, 2012; A, left lateral view of complete carapace, P6M3742, 11CHAO80; B, right lateral view of complete carapace, P6M3743, 11CHAO80; C, Knoxitidae sp. 1, right lateral view of complete carapace, P6M3744, sample 11CHAO171; D, Knoxiella sp. 1, right lateral view of complete carapace, P6M3745, sample 11CHAO156; E, F, Knoxiella sp. 2; E, left lateral view of complete carapace, P6M3746, sample 11CHAO80; F, right sublateral view of complete carapace, P6M3747, sample 11CHAO80; G, Permoyoungiella? sp. 1, right lateral view of right valve, P6M3748, sample 11CHAO172; H, Kirkbyoidea sp. 1, left lateral view of left valve, P6M3749, sample 11CHAO166; I, Kirkbyoidea sp. 2, left lateral view of left valve, P6M3750, sample 11CHAO168; J, Kirkbyoidea sp. 3, right lateral view of right valve, P6M3751, sample 11CHAO167; K, L, Roundyella? sp. 1; K, left lateral view of left valve, P6M3752, sample 11CHAO168; L, left lateral view of complete carapace, P6M3753, sample 11CHAO168; M, Roundyella sp. 2, left lateral view, P6M3754, sample 11CHAO158; N, Knightina sp. 1, right lateral view of right valve, P6M3755, sample 11CHAO173; O, Knightina? sp. 2, right lateral view of right valve, P6M3756, sample 11CHAO167. Scale bar: $100 \mu \mathrm{m}$. 
Samarella viscusforma Chitnarin, 2012

(Fig. 16M, N)

Samarella viscusforma Chitnarin in Chitnarin et al., 2012: 818, 819, figs 13A-E, 15.

MATERIAL EXAMINED. - 34 complete carapaces and eight broken carapaces.

Dimensions. $-\mathrm{L}=281-592 \mu \mathrm{m} ; \mathrm{H}=193-477 \mu \mathrm{m}$.

OCCURRENCES. - Loei Province, Northeastern Thailand, Early Permian, Phetchabun Province, Central Thailand, Early Permian and Nakhon Sawan Province, Central Thailand, Late Early Permian and Middle Permian (Chitnarin et al. 2012); Chaotian section, Sichuan Province, China, Maokou and Wujiaping Formations, Middle Capitanian-basal Wuchiapingian, Middle-Late Permian (this study, samples: 11CHAO 173, 11CHAO172, 11CHAO171, 11CHAO 169, 11CHAO 163, 11CHAO 151, 11CHAO 80, 11CHAO81, 11CHAO82, 11CHAO83, 11CHAO84, 11CHAO88, 11CHAO92, 11CHAO100, 11CHAO101, see Table 1).

\section{REMARKS}

Variations could be quite important in carapace size, DB shape or VB convexity. The question of the presence of different species could come up. But we observe all the transitional forms between the different extreme morphotypes. So we make the choice to gather all of them in S. viscusforma.

Order PLATYCOPIDA Sars, 1866

Suborder PLATYCOPINA Sars, 1866

Superfamily CAVELLINOIDEA Egorov, 1950

Family CAVELLINIDAE Egorov, 1950

Genus Sulcella Coryell \& Sample, 1932

TyPe SPECIES. - Sulcella sulcata Coryell \& Sample, 1932 by original designation.

Sulcella mesopermiana? Kozur, 1985

(Fig. 17A)

Sulcella mesopermiana Kozur, 1985: 22, pl. 5, figs 3, 4. —CrasquinSoleau \& Baud 1998: pl. 3, figs 3, 5, 6, 9.

MATERIAL EXAMINED. - One complete carapace.

Dimensions. $-\mathrm{L}=759 \mu \mathrm{m} ; \mathrm{H}=434 \mu \mathrm{m}$.

OCCurrences. - Bükk Mountains, Hungary, Middle and Late Permian (Kozur 1985); Hydra Island, Greece, Late Permian (Crasquin-Soleau \& Baud 1998); ?Chaotian section, Sichuan Province, China, Wujiaping Formation, basal Wuchiapingian, Late Permian (this study, samples: 11CHAO88, see Table 1).

\section{REMARKS}

Our specimen is very similar to $S$. mesopermiana Kozur, 1985 from Middle-Late Permian of Hungary and Greece (Kozur 1985) but differs in having longer and horizontal DB, shorter $\mathrm{PDB}$ and more arched PB.
Sulcella suprapermiana? Kozur, 1985

(Fig. 17B, C)

Sulcella suprapermiana Kozur, 1985: 22, pl. 5, figs 6, 8. - CrasquinSoleau \& Baud 1998: pl. 4, figs 1, 3.

MATERIAL EXAMINED. - Two complete carapaces.

Dimensions. $-\mathrm{L}=624-665 \mu \mathrm{m} ; \mathrm{H}=285-313 \mu \mathrm{m}$.

OCCURRENCES. - Bükk Mountains, Hungary, Late Permian (Kozur 1985); Hydra Island, Greece, Late Permian (Crasquin-Soleau \& Baud 1998); ?Chaotian section, Sichuan Province, China, Maokou and Wuchiaping Formations, Middle Capitanian-basal Wuchiapingian, Middle-Late Permian (this study, samples: 11CHAO166, 11CHAO81, see Table 1).

\section{REMARKS}

Our specimens are very similar to $S$. suprapermiana Kozur, 1985 from Late Permian of Hungary and Greece (Kozur 1985; Crasquin-Soleau \& Baud 1998) but differ in having nearly vertical PB.

\section{RESULTS}

\section{SPECIES DISTRIBUTION}

Study of the 40 fossiliferous samples from the Chaotian section allowed us to identify 154 ostracod species belonging to 29 genera, 16 families, 12 superfamilies and three orders. Assemblages present a relatively high diversity at high taxonomic levels. Thirty-seven of the analysed samples yield identifiable specimens. The species distribution is presented in Table 1.

Capitanian samples record 61 species. Most of them seem to disappear gradually by the top of the L3 subunit. The M1 subunit yields only five identified species. Transition from Capitanian to Wuchiapingian, at specific level, is marked by a near complete renewal of assemblages. Only four species, presenting a rather long stratigraphic range, passed the GLB. The transition is furthermore characterised by a significant increase of diversity after GLB with 96 species recorded in Wuchiapingian samples. Thus, specific extinction rate at the GLB is about $93 \%$ and turnover rate is about $97 \%$.

However, variations at higher taxonomic levels are quite dissimilar. From the 25 genera recorded in Capitanian samples, only 16 pass the GLB. Four genera are here found only in the Wuchiapingian. It corresponds to a "disappearance" of about $36 \%$ of genera and a $20 \%$ turnover rate. From the 16 families found in Capitanian samples, 13 are also present in the Wuchiapingian. Kellettinidae, Scorbiculidae and Youngiellidae seem to "disappear". Nevertheless these three families are known in the Late Permian from other localities, even though they are clearly in decline. Scrobiculidae are for example found, mainly represented by the genus Roundyella, in the Wuchiapingian of Hungary (Kozur 1985) and Iran (Mette 2008) and the Changhsingian of Israel (Gerry et al. 1987). Kellettinidae are known in the Wuchiapingian (i.e. genus Kindlella in Hungary - Kozur 1985) and the Changhsingian (i.e. genus Kellettina in South China - Yuan et al. 2007). Youngiellidae occur in the Wuchiapingian (for example with 
A

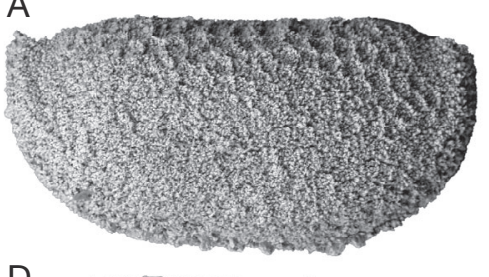

B

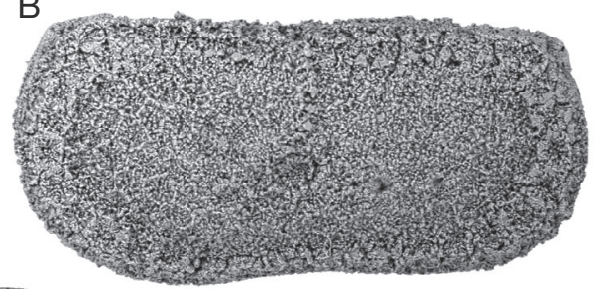

C

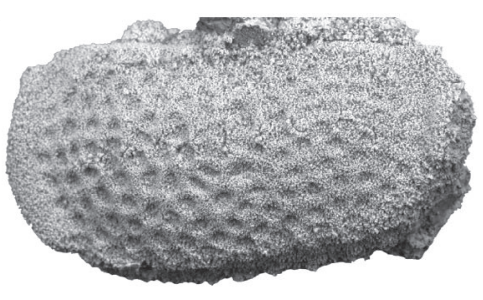

D

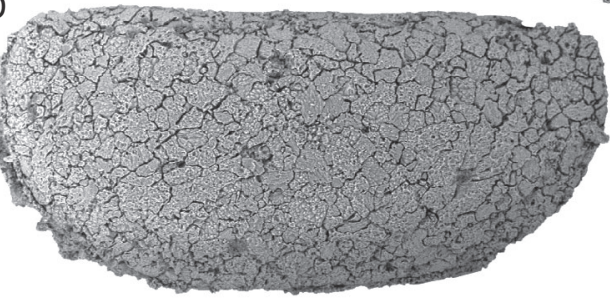

E
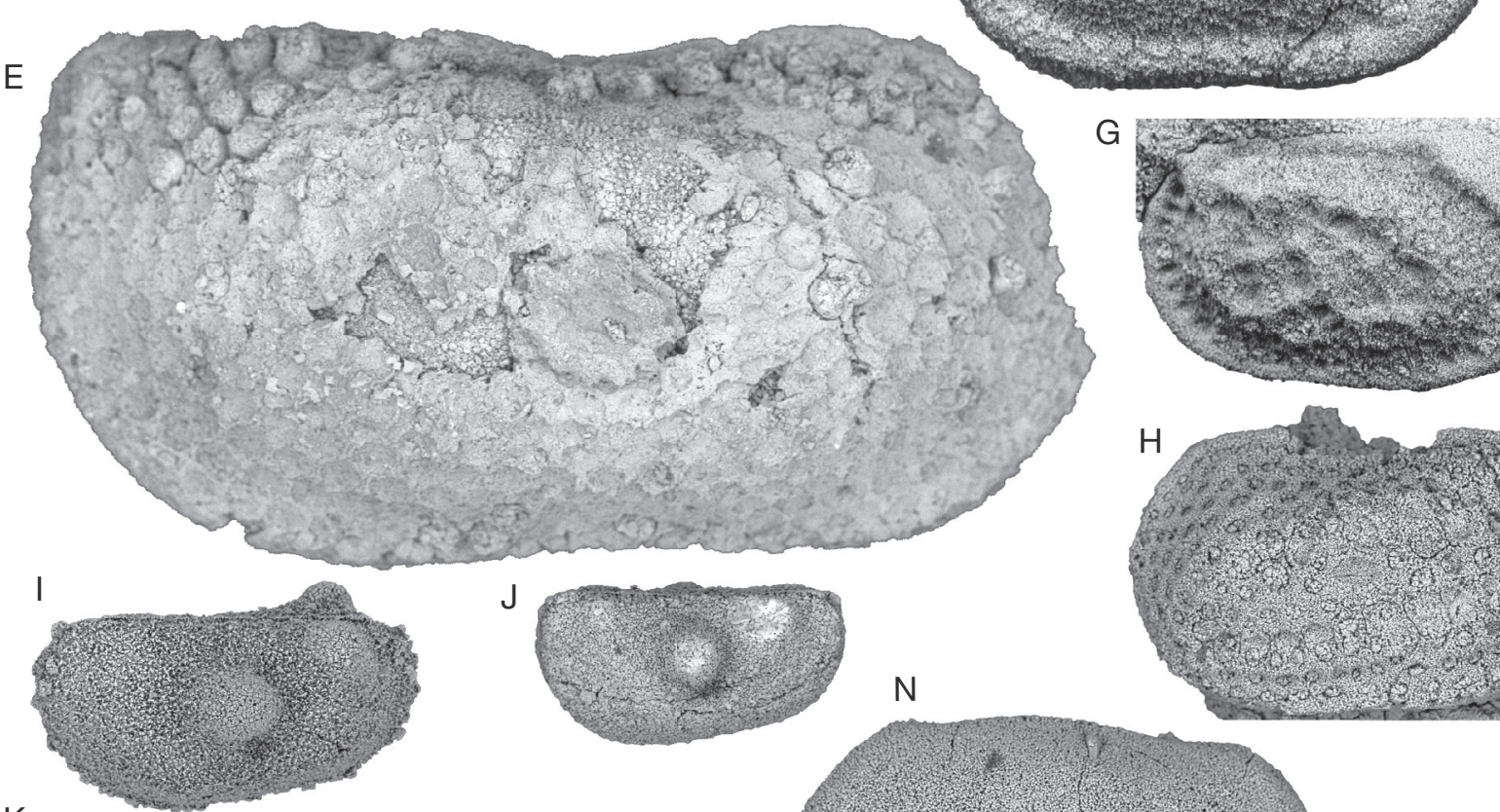

K
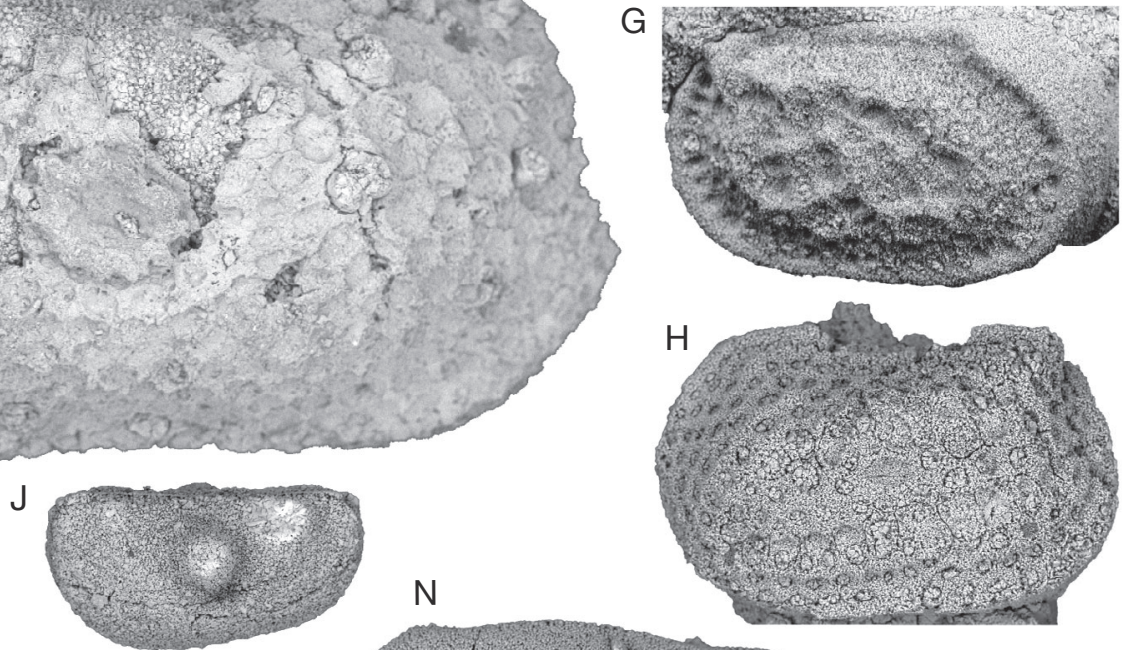

N

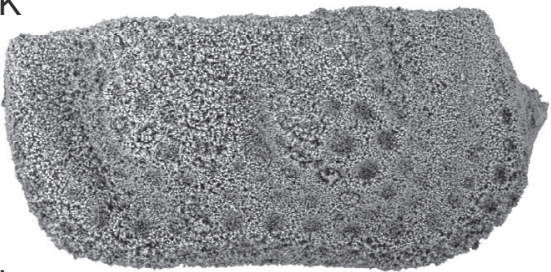

L
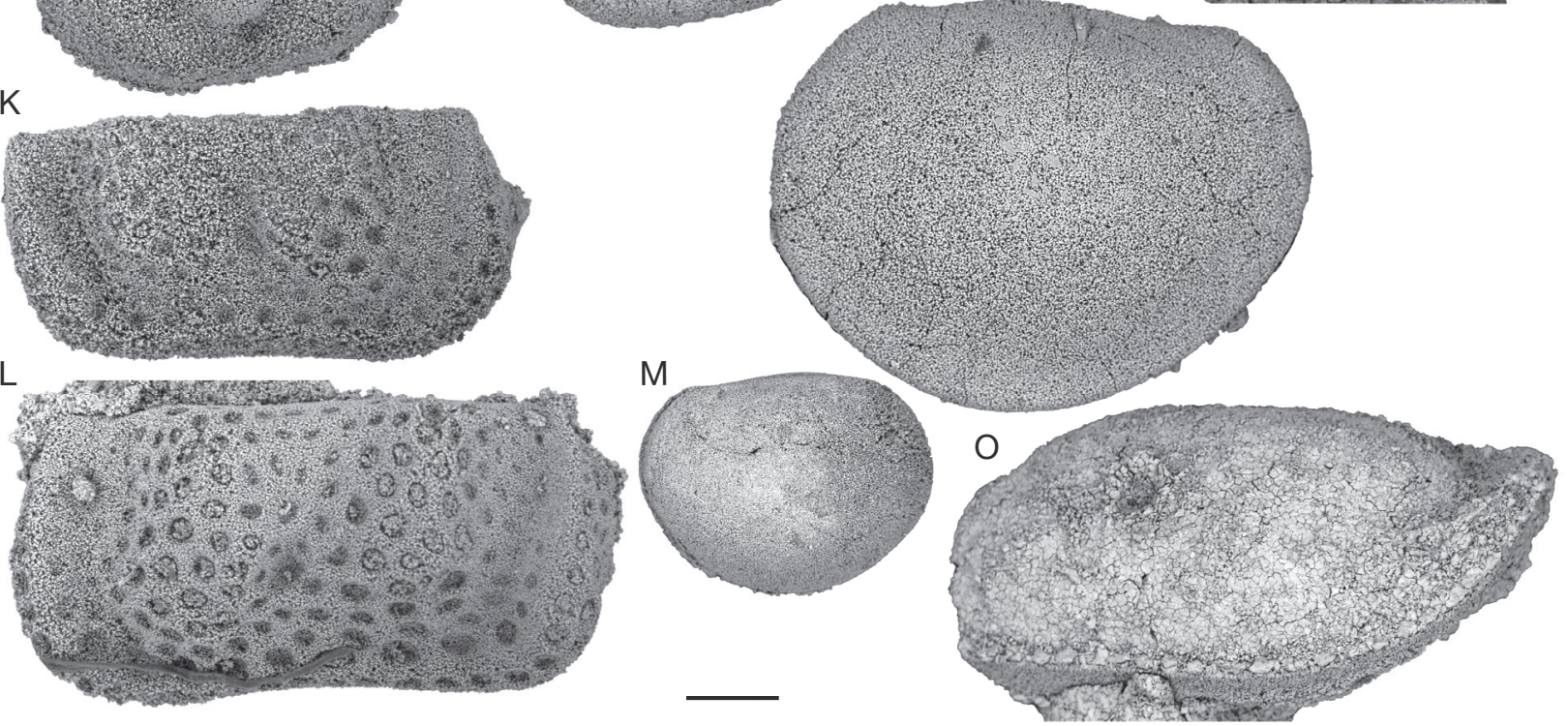

FIG. 16. - Ostracods from Guadalupian-Lopingian boundary interval in South China: A, Kirkbya sp. 1, right lateral view of complete carapace, P6M3757, sample 11CHAO172; B, Kirkbya sp. 2, left lateral view of left valve, P6M3758, sample 11CHAO164; C, Kirkbya sp. 3, left lateral view of left valve, P6M3759, sample 11CHAO166; D, Kirkbya sp. 4, left lateral view of left valve, P6M3760, sample 11CHAO158; E, Kirkbya sp. 5, left lateral view of left valve, P6M3776, sample 11CHAO88; F, G, Reviya cf. subsompongensis Chitnarin, 2008; F, right lateral view of right valve, P6M3762, sample 11CHAO167; G, right lateral view of right valve, P6M3763, sample 11CHAO166; H, Neoamphissites sp. 1, left lateral view of left valve, P6M3764, sample 11CHAO169; I, J, Kegelites sp. 1; I, left lateral view of complete carapace, P6M3765, sample 11CHAO81; J, left lateral view of complete carapace, P6M3766, sample 11CHAO99; K, L, Kindlella sp. 1; K, right lateral view of complete carapace, P6M3767, sample 11CHAO150; L, left lateral view of complete carapace, P6M3768, sample 11CHAO150; M, N, Samarella viscusforma Chitnarin, 2012; M, right lateral view of complete carapace, P6M3769, sample 11CHAO88; N, left lateral view of complete carapace, P6M3770, sample 11CHAO171; O, Kirkbya sp. 6, sublateral view of broken left valve, P6M3761, sample 11CHAO97. Scale bar: 100 um. 


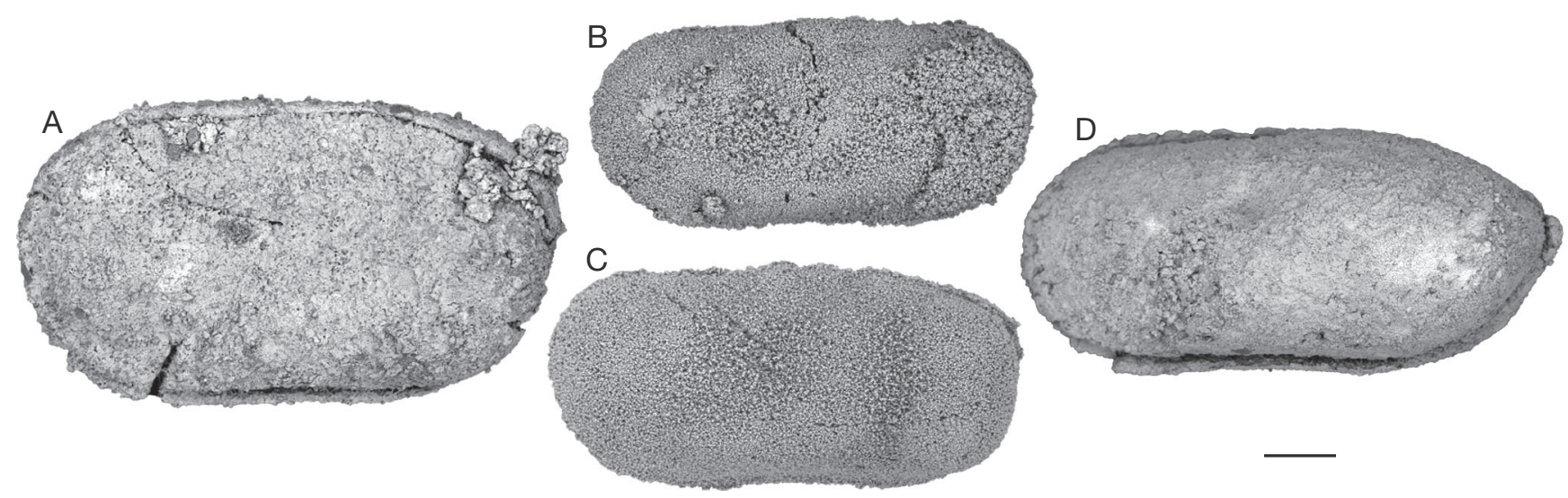

FIG. 17. - Ostracods from Guadalupian-Lopingian boundary interval in South China. A, Sulcella mesopermiana? Kozur,1985, P6M3771, 11CHAO88; B, C, Sulcella suprapermiana? Kozur,1985; B, P6M3772, 11CHAO166; C, P6M3773, 11CHAO81; D, Sulcella sp. 1, P6M3774, sample 11CHAO88. All photographs represent complete carapaces in left lateral view. Scale bar: $100 \mu \mathrm{m}$.

Permoyoungiella in Hungary - Kozur 1985 or Youngiella in Iran - Mette 2008) and in the Changhsingian (for example Permoyoungiella in China - Crasquin et al. 2010 or Moorites in Israel - Gerry et al. 1987).

\section{OSTRACOD ABUNDANCE AND DIVERSITY}

Ostracods are present from the upper part of the Maokou Fm "Limestone Unit" (L3 subunit, sample 11CHAO173, Jinogondolella postserrata conodont Zone) up to the first $27 \mathrm{~m}$ of the Wujiiaping Formation (11CHAO101, CodonofusiellaReichelina small fusuline assemblage), except in the middle part of the M1 subunit (see Material and methods).

In productive samples, abundance varies from one (11CHAO74) to 366 specimens (and 1895 undetermined fragments, 11CHAO88) and specific richness from one (11CHAO74) to 44 (11CHAO95) species. In most of assemblages the specific richness variations correlates with abundance (Fig. 20).

Ostracod faunas, from the L3 subunit of the Maokou Fm "Limestone Unit", show relatively high abundance and specific richness (11CHAO171, 361 specimens, 549 undetermined fragments; 11CHAO173, 32 species). Nevertheless, a two-step decrease of both abundance and specific richness is recorded through this interval until the "disappearance" of ostracods at the top of the "Limestone Unit" (11CHAO152).

The two productive samples of the M1 subunit (11CHAO 156 and 158, corresponding to the only two carbonate beds in the "Mudstone Unit") record relatively low abundance and diversity assemblages.

Basal six Wuchiapingian samples present the lowest abundance and specific richness recorded in the section. Indeed, except the very first Wuchiapingian sample (11CHAO72, 18 specimens belonging to one species, 11 undetermined fragments), the above samples yield only zero to six specimens (zero to 23 undetermined fragments) and no more than one identified species.

Finally, ostracod fauna recovery begins in the Wujiaping Fm around three meters above the GLB. It is marked by a two-step increase of abundance and diversity. Maximum
Wuchiapingian abundance value (11CHAO88, 366 specimens, 1895 undetermined fragments) reaches the Capitanian one. The maximum of specific richness is substantially higher than Capitanian one, with the highest values recorded in the section (11CHAO95, 44 species).

Ostracods identified in Chaotian section samples belong to three orders: Podocopida, Palaeocopida and Platycopida. Palaeocopida are known to progressively disappear during the Late Permian, to become extinct after the PTB (Permian-Triassic Boundary, Crasquin-Soleau et al. 2004; Crasquin \& Forel 2014). Thus, study of the distribution variations at order level at the GLB could bring relevant information about changes in ostracod assemblages during this poorly known period. The Figure 20 illustrates diversity and abundance variations of Podocopida and Palaeocopida along the section. Because of the too low number of Platycopida specimens found, their abundance and diversity variations are not figured here.

The Order Podocopida is the most abundant in Chaotian samples and represents $92.6 \%$ of identifiable specimens. It also presents the highest specific richness with 125 species identified corresponding to $80 \%$ of the total species recorded. These species belong to 13 genera, six families, five superfamilies and three suborders. Capitanian samples record 40 Podocopida species (Table 1). Only two of these are also found in Wuchiapingian samples, belonging to two distinct genera (Bairdia and Microcheilinella). The specific extinction rate is about $95 \%$. Wuchiapingian samples record 86 species, twice more than Capitanian ones. Specific turnover rate is about $98 \%$.

As they are largely dominant in samples, Podocopida abundance and specific richness variation curves follow the curves of complete assemblages (Fig. 20). Thus, high values recorded in basal samples from the upper L3 subunit (11CHAO171, 340 specimens, 26 species) also present a two-step decrease until ostracod "disappearance" at the top of L3 subunit.

The M1 subunit and the base of the Wujiaping Fm are characterised by a very low abundance and diversity phase (Table 1; Fig. 20). 
The two-step "recovery", previously evoked, is also noticeable here. If maximum Wuchiapingian abundance values here reached (11CHAO88, 357 specimens) are similar to Capitanian maxima, highest Wuchiapingian specific richness values (11CHAO95, 42 species) are almost twice as great as Capitanian ones.

The Order Palaeocopida is significantly less abundant in samples with $7.2 \%$ of specimens and $18 \%$ of species (27 species). Nevertheless, it presents a relatively high generic and family level diversity with 15 genera (51.8\%) and nine families (56.3\%). Four Palaeocopida superfamilies belonging to two sub-orders are represented at Chaotian. Capitanian samples record 20 Palaeocopida species (Table 1). Only one of these is still present in Wuchiapingian samples, belonging to genus Samarella. The specific extinction rate is about $95 \%$. Wuchiapingian samples record only eight species. Specific turnover rate is about $87.5 \%$.

Much less abundant than Podocopida, Palaeocopida present moreover quite dissimilar distribution variations along the Chaotian section. If Capitanian abundance and specific richness are also maximum at the base of the upper L3 subunit (11CHAO173, 26 specimens, eight species), the extinction pattern differs from Podocopida. Indeed, relatively high values are also found in the middle part of this interval (11CHAO166,

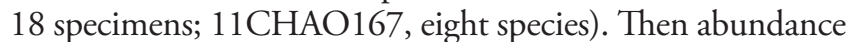
and specific richness decrease sharply until the "disappearance" of ostracods at the top of the L3 subunit (11CHAO163).

Unlike Podocopida, the two fossiliferous samples from the M1 subunit present here relatively high abundance values (11CHAO158, 14 specimens) but a low specific diversity (maximum two species, 11CHAO158).

The very first Wuchiapingian sample presents a relatively high abundance (18 specimens belonging to Hollinella genus, study in progress). Basal Wuchiapingian samples record extremely low abundance and specific richness.

Values rise rapidly to a Wuchiapingian maximum (11CHAO80) with a relatively high abundance (20 specimens) but specific richness stay really poor (three species). From that point, abundance and specific richness finally decrease and stabilise at relatively low values (zero to five specimens, zero to two species) to the top of the section.

Thus, the early Late Permian (Wuchiapingian) is here characterised by abundance and specific diversity significantly lower than those recorded in the late Middle Permian (Early/ Middle Capitanian).

The Order Platycopida is the less abundant one in Chaotian samples with only seven specimens $(0.2 \%)$, three species $(2 \%)$ belonging to one genus (Sulcella). Due to the very occasional occurrences of specimens, we have not been able to distinguish any particular trend in Platycopida distribution along Chaotian section.

\section{DISCUSSIONS}

\section{PalaeoenVironmental CHANGes AT GLB}

The L3 subunit from the "Limestone Unit" of the Maokou Fm is commonly associated with shallow-marine environment with sediments deposited on a continental shelf probably

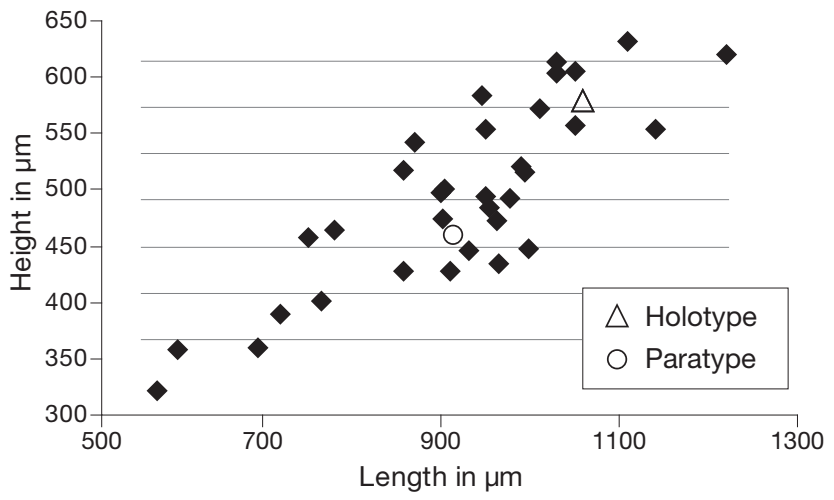

FIG. 18. - Height-length diagram of Bairdia chaotianensis Zazzali, n. sp.

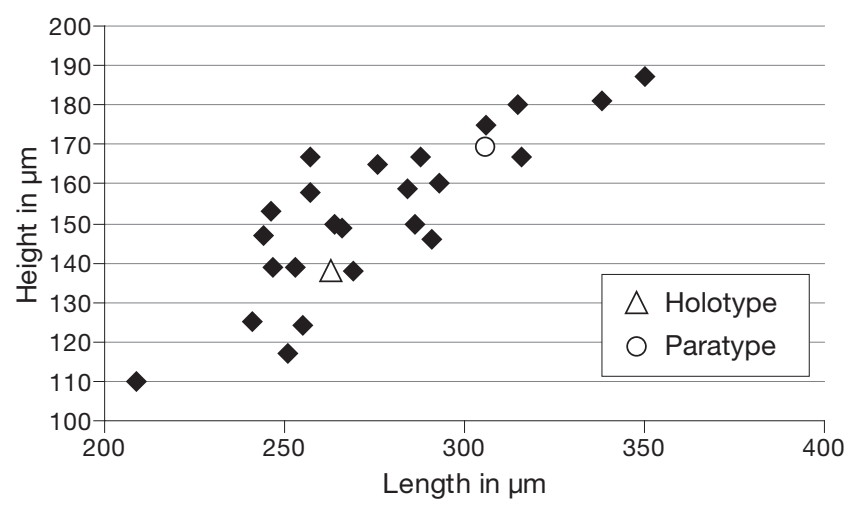

FIG. 19. - Height-length diagram of Microcheilinella pagodaensis Zazzali, n. sp.

below storm wave base, within the euphotic zone (Isozaki et al. 2008; Lai et al. 2008; Saitoh et al. 2013a). While interpretations of these deposits seem to be a consensus, the top of the L3 subunit is the starting point of divergence between those suggesting a regression phase (Lai et al. 2008) and those proposing a transgression phase (Isozaki et al. 2008; Saitoh et al. 2013a). Lai et al. (2008) evoke an increasing degree of fragmentation of the bioclasts towards the L3 subunit, which could be in agreement with the gradual shallowing hypothesis. However, the fragment abundance does not seem to increase in our samples. In the top $5 \mathrm{~m}$ of the L 3 subunit, Isozaki et al. (2008) evoke a decrease of granulometry coincident with the beginning of a transgression phase. If our study could not allow, for now, to discuss about that last interpretation, we could nevertheless notice that this interval correspond to the decreasing trend of ostracod abundance and specific richness.

The "Mudstone Unit" is also a subject of controversy, presented as lagoonal deposit by Lai et al. (2008) and as deep sea deposit by Isozaki et al. (2008) and Saitoh et al. (2013a). Basal samples of the M1 subunit are barren of ostracods. Lai et al. (2008) characterised this interval by a disappearance of shallow marine taxa at the base of the "Mudstone Unit" (M1) due to a lagoonal hypersaline environment and by the presence of brachiopods at the top of the M2 subunit testifying more open-marine connections.

In our study, the ostracods of the M1 subunit are marine taxa. Furthermore, brachiopods were observed on the field 
both in M1 and M2 subunits during our sampling (Fig. 4). This invalids the hypothesis of a lagoonal environment. Isozaki et al. (2008) and Saitoh et al. (2013a) evoke a basal subunit (M1) yielding abundant shallow marine taxa, such as conodonts, brachiopods, small foraminifera and ostracods and deposited in a first deepening phase. These taxa seem to have disappeared from the M2 subunit, replaced by deep marine taxa such as ammonoids and radiolarians, also with brachiopods in some beds. The ostracod associations in the two productive samples of the "Mudstone Unit" are poorly preserved but are clearly marine neritic taxa, with genera (Kirkbya, Roundyella, Knoxiella) characteristic of a shallow environment (Melnyk \& Maddocks 1988a).

The hypothesis of a regression, during the missing Late Capitanian at Chaotian (Saitoh et al. 2013a), leading to an emergence phase at the top of the Wangpo Bed, is commonly accepted.

The basal part of the Wujiaping Fm is commonly presented as deposited during a rapid transgressive phase to a shallow marine environment. Samples from the base of the Wujiaping Fm yielded very few ostracods. Above the very first Wuchiapingian sample, this low abundance phase could be linked with rapid environmental changes, during a quick transgression, as proposed in the literature.

As the "Limestone Unit" of the Maokou Fm, the Wujiaping Fm is commonly associated with shallow-marine environment (Isozaki et al. 2008; Lai et al. 2008; Saitoh et al. 2013a). While further analyses of ostracods assemblages are needed in order to achieve precise palaeoenvironmental reconstructions, the prompt ostracod diversity recovery observed in the Wujiaping Fm suggests a rapid reinstatement of stable environmental conditions.

\section{FAUNAL CHANGES}

Transition from Guadalupian to Lopingian, at Chaotian section seems coincident with important faunal changes. The passage from the "Limestone Unit" to the "Mudstone Unit", and then to the Wujiaping Fm, is marked by noticeable losses and appearances. Thus, high fusuline diversity, with presence of large forms (Neoschwargerina, Lepidolina, Isozaki et al. 2008) gives away to exclusively small form assemblages in the M2 subunit and in the Wujiaping Fm (Lai et al. 2008). Robust forms of calcareous algae, recorded in Capitanian, are replaced by more fragile forms at the base of Wuchiapingian (Lai et al. 2008). Crinoids, rugose corals and bivalves seem to disappear at the top of the L3 subunit (Isozaki et al. 2008; Saitoh et al. 2013a). Conodont last appearance is located in the "Mudstone Unit" (Isozaki et al. 2008), where taxa such as ammonoids and radiolarians are exclusively found. Then, diversity decreases radically in the first meters of the Wujiaping Fm.

Ostracod assemblages seem also clearly affected, from the Early-Middle Capitanian (L3) to the basal Wuchiapingian, by a significant decrease of abundance and diversity. If initial abundance seems to recover quickly after the GLB, it is important to notice that specific richness became even more important in Wuchiapingian records. Moreover, data present an important renewal in this interval (93\% extinction rate and $96 \%$ turnover rate).
Transition from the Early-Middle Capitanian to the Late Permian seems to affect differently the Orders Podocopida and Palaeocopida.

Podocopida abundance and diversity decrease during Early Capitanian (L3 subunit), becoming extremely low in the EarlyMiddle Capitanian (M1 subunit) and the basal Wuchiapingian. However, recovery is recorded quite early in the Wuchiapingian sequence and specific richness reaches values twice as great as the highest ones recorded in the Capitanian. Thus, post-GLB assemblages seem more diversified.

Palaeocopida abundance also decreases at the top of the L3 subunit, but it remains relatively high in the M1 subunit fossiliferous samples and in the first Wuchiapingian sample. Specific richness decreases promptly from the middle to the top of the upper L3 subunit, not to rise significantly again. Coincidently with the Podocopida recovery, Palaeocopida abundance decreases a second time to remain low up to the top of the section. In any case, post-GLB Palaeocopida assemblages seem less diversified.

Palaeocopida are known to disappear progressively in the Late Permian, to become extinct at the basal Middle-Triassic (Crasquin-Soleau et al. 2004; Forel \& Crasquin 2011; Crasquin \& Forel 2014), while Podocopida became the predominant order during the Mesozoic-Recent period. Palaeocopida response to GLB events might here be interpreted as the first step of their early Middle-Triassic disappearance. Although this hypothesis seems in agreement with the GLB extinctions evoke in literature, additional investigations and more data from Late Capitanian (missing in this section) and from the Wujiaping Fm are needed. Data obtained in this study might, however, be interpreted as resulting from temporary and/or regional environmental changes, in agreement with the generic and family level "disappearances" recorded. Thus, these changes might have led to the establishment of environmental conditions more favourable to Podocopida than to Palaeocopida, as they present different patterns at the base of Wuchiapingian.

Further palaeoenvironnemental reconstitutions based on ostracod assemblage analysis, in addition to comparison with data from palaeogeographically distant sections, such as Penglaitan GSSP (South China) or Iran (both work in progress), will bring essential information to test these hypotheses.

\section{Acknowledgements}

Sindbad Zazzali was supported by a fellowship from the Ministère de l'Enseignement Supérieur et de la Recherche, France. Field trip was realized with the support of the Chinese 111 program (B08030) and the Ministry of Education of China (20110145130001).

We thank Martine Fordant (UMPC) for her help in sample processing and picking of ostracods and Alexandre Lethiers (UPMC) for illustration realisation. We are grateful to Dr Anisong Chitnarin (School of Geotechnology, Institute of Engineering, Suranaree University of Technology, Thailand) for her help and discussion on systematic comparisons and Dr Yuan Aihua (Faculty of Earth Sciences, China University of Geosciences, Wuhan, P. R. China) for her help in translating descriptions from Chinese. 


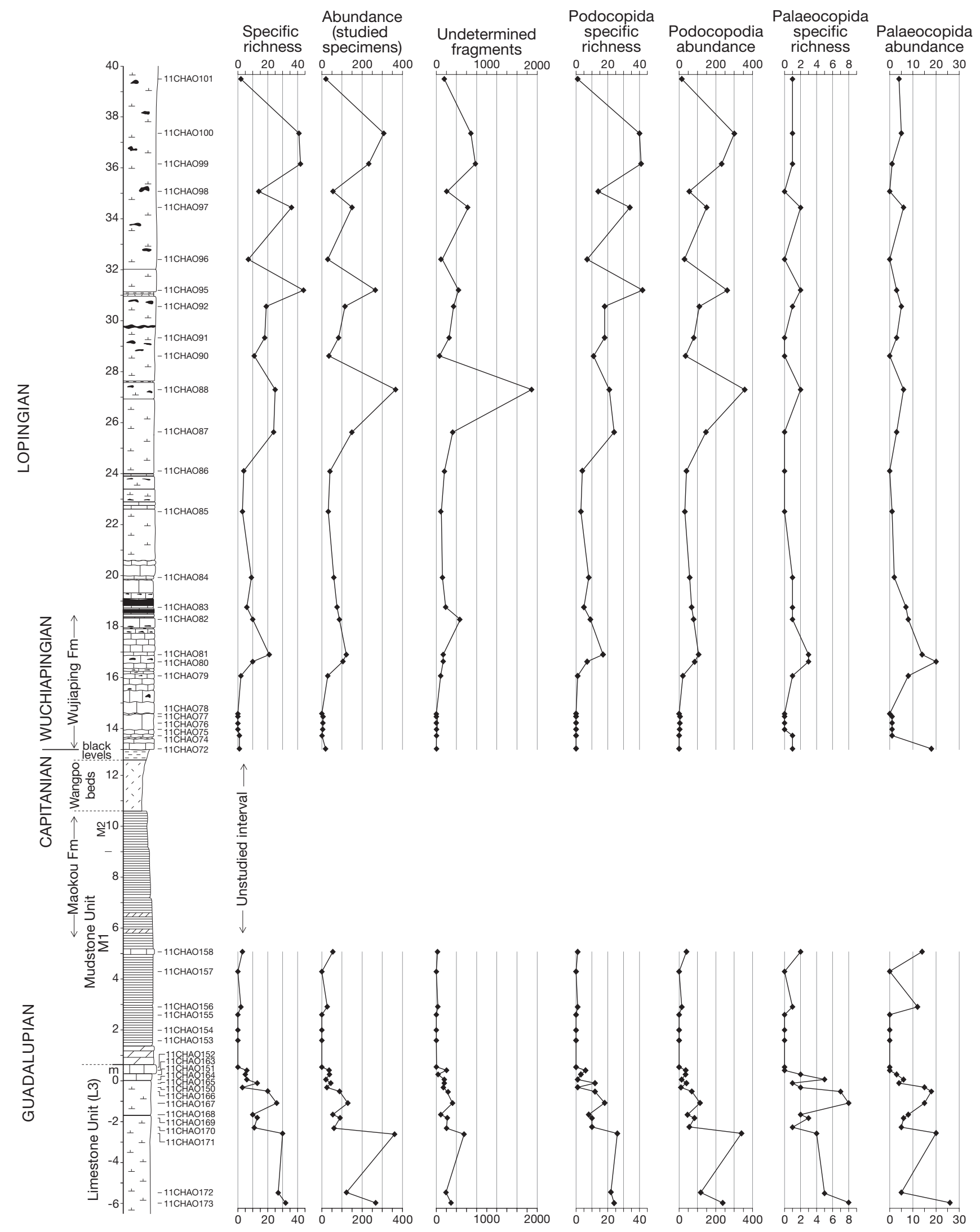

FIG. 20. - Specific richness (number of species), abundances (number of specimens) and number of unidentified fragments through Chaotian section. For lithology, same caption as Figure 4. 
We finally thank reviewers, Dr Avraham Honigstein (Ministry of National Infrastructures Oil and Gas section, Jerusalem, Israel) and Pr. David J. Horne (Queen Mary University, London, United Kingdom) for their reviewing and their help to improve our manuscript.

\section{REFERENCES}

Bond D. P. G., Wignall P. B., Wang W., IzON G., Jiang H. S., Lai X. L., Sun, Y. D., Newton R. J., Shao L. Y., Védrine S. \& Cope H. 2010a. - The mid-Capitanian (Middle Permian) mass extinction and carbon isotope record of South China. Palaeogeography, Palaeoclimatology, Palaeoecology 292: 282-294.

Bond D. P. G., Hilton J., Wignall P. B., Ali J. R. \& Stevens L. G. 2010b. - The Middle Permian (Capitanian) mass extinction on land and in the oceans. Earth-Science Reviews 102: 100-116.

BurReTt C., UdCHACHON M., ThaSSANAPAK H. \& CHITNARIN A. 2014. - Conodonts, radiolarians and ostracodes in the Permian E-Lert Formation, Loei Fold Belt, Indochina Terrane, Thailand. Geological Magazine: 1-37.

Chen T. C. 1958. - Permian ostracods from the Chihsia limestone of Lungtan, Nanking. Acta Palaeontologica Sinica 6: 215-257.

Chen D. \& SHI C. 1982. — Latest Permian ostracoda from Nantong, Jiangsu and from Miannyang, Hubei. Bulletin of Nanjing Institute of Geology and Palaeontology, Academic Sinica 4: 105-152.

CHen D. \& BAO H. 1986. - Lower Permian ostracodes from the Chihsia Formation of Jurong and Longtan, Jiangsu province. Acta Micropalaeontologica Sinica 3: 107-132.

ChITNARIN A. 2010. - Taxonomy of Permian Ostracods from Central, Northeastern, and Western Thailand: Implications for Paleoenvironment and Paleobiogeography. PhD Thesis, Suranaree University of Technology, Nakhon Ractchasima, Thailand, 190 p.

Chitnarin A., Crasquin S., Chonglakmani C., Broutin J., Grote P. J. \& Thanee N. 2008. — Middle Permian ostracods from Tak Fa Limestone, Phetchabun Province, Central Thailand. Geobios 41: 341-353.

Chitnarin A., Crasquin S., Charoentitirat T., Tepnarong P. \& ThaneE N. 2012. — Ostracods (Crustacea) of the Early-Middle Permian from Central Thailand (Indochina block). Part 1. Order Palaeocopida. Geodiversitas 34 (4): 801-835. http://dx.doi. org/10.5252/g2012n4a5

Clapham M. E., Shen S. Z. \& BotTjer D. J. 2009. - The double mass extinction revisited: reassessing the severity, selectivity, and causes of the end-Guadalupian biotic crisis (Late Permian). Paleobiology 35 (1): 32-50.

CORDELL R. J. 1952. - Ostracodes from the Upper Pennsylvanian of Missouri. Part I. The Bairdiidae. Journal of Paleontology 26: 74-112.

CRASQuin S. \& Forel M. B. 2014. - Ostracods (Crustacea) through Permian-Triassic events. Earth-Science Reviews 137: 52-64.

Crasquin S., Forel M. B., Feng Q., YuAN A., Baudin F. \& Collin P.-Y. 2010. - Ostracods (Crustacea) through Permian-Triassic boundary in SouthChina: the Meishan stratotype (Zhejiang Province). Journal of Systematic Palaeontology 8: 331-70.

Crasquin-Soleau S. \& Baud A. 1998. - New Permian ostracods from Greece (Hydra Island). Journal of Micropalaeontology $17: 131-52$.

Crasquin-Soleau S., Marcoux J., Angiolini L. \& Nicora A. 2004. - Palaeocopida (Ostracoda) across the Permian-Triassic events: new data from southwestern Taurus (Turkey). Journal of Micropalaeontology 23: 67-76.

Crasquin-Soleau S., Vaslet D. \& Le Nindre Y. M. 2005. Ostracods from Permian-Triassic boundary in Saudi Arabia (Khuff Formation). Palaeontology 48: 853-868.

Deconinck J. F., Crasquin S., Bruneau L., Pellenard P., Baudin F. \& FENG Q. 2014. — Diagenesis of clay minerals and K-benton- ites in the Late Permian/Early Triassic sediments of the Sichuan Basin (Chaotian section, Central China). Journal of Asian Earth Sciences 81: 28-37.

Forel M. B. \& CRASQuin S. 2011. - In the aftermath of the Permian-Triassic boundary mass extinction: new ostracod (Crustacea) genus and species from south Tibet. Geodiversitas 33 (2): 247-263. http://dx.doi.org/10.5252/g2011n2a3

Forel M. B., Crasquin S., Hips K., Kershaw S., Collin P.-Y. \& HAAS J. 2013. - Biodiversity evolution through the PermianTriassic boundary event: Ostracods from the Bükk Mountains, Hungary. Acta Palaeontologica Polonica 58 (1): 195-219.

Gerry E., Honigstein A., Derin B. \& FleXer A. 1987. — Late Permian Ostracodes of Israel. Taxonomy, distribution and paleogeographical implications. Senckbergiana lethaea 68 (1/4): 197-223.

GolONKA J. 2002. - Plate-tectonic maps of the Phanerozoic, in Kiessling W., FlÜgel E., \& Golonka J. (eds), Phanerozoic Reef Patterns. SEPM Special Publication 72: 21-76.

Guan S., Sun Q., Jiang Y., Li L., Zhao B., Zhang X., Yang R. \& Feng B. 1978. - Subclass ostracoda. In Paleontological Atlas of Central and South China. Geological Publishing House, Beijing: 115-325.

He B., Xu Y-G., Huang X-L., LuO Z-Y, SHI Y-R., YAN, Q. J. \& YU S-Y. 2007. - Age and duration of the Emeishan flood volcanism, SW China: geochemistry and SHRIMP zircon U-Pb dating of silicic ignimbrites, post-volcanic Xuanwei Formation and clay tuff at the Chaotian section. Earth and Planetary Science Letters 255: 306-323.

He B., Xu Y. G., Zhong Y. T. \& GUAN J. P. 2010. - The Guadalupian-Lopingian boundary mudstones at Chaotian (SW China) are clastic rocks rather than acidic tuffs: Implication for a temporal coincidence between the end-Guadalupian mass extinction at the Emeishan volcanism. Lithos 119: 10-19.

Horne D. J., Cohen A. \& Martens K. 2002. - Taxonomy, Morphology and Biology of Quaternary and living Ostracoda, in HOlmes J. A. \& ChIVAS A. (eds), The Ostracoda: Applications in Quaternary Research. Geophysical Monograph, American Geophysical Union, Washington DC: 5-36.

IsHIZAKI K. 1964. - Middle Permian ostracodes from the Iwaizaki Limestone, Northeast Japan. Science Reports of the Tohoku University, Second Series (Geology) 36: 139-160.

ISOZAKI Y. 2009. - Integrated "plume winter" scenario for the double-phase extinction during the Paleozoic-Mesozoic transition: The G-LB and P-TB events from a Panthalassan perspective. Journal of Asian Earth Sciences 36: 459-480.

IsOZAKI Y. \& ALJINOVIC D. 2009. — End-Guadalupian extinction of the Permian gigantic bivalve Alatoconchidae: end of gigantism in tropical seas by cooling. Palaeogeography, Palaeoclimatology, Palaeoecology 284: 11-21.

ISOZAKI Y., YAO J. X., MATSUdA T., SAKAI H., Ji Z. S., SHIMIZU N., Kobayashi N., Kawahata H., Nishi H., Takano M. \& Kubo T. 2004. - Stratigraphy of the Middle-Upper Permian and Lowermost Triassic at Chaotian, Sichuan, China - record of Late Permian double mass extinction event. Proceedings of Japan Academy, Series B 80: 10-16.

Isozaki Y., Shimizu N., Yao J., Ji Z. \& Matsuda T. 2007. — EndPermian extinction and volcanism-induced environmental stress: The Permian-Triassic boundary interval of lower-slope facies at Chaotian, South China. Palaeogeography, Palaeoclimatology, Palaeoecology 252: 218-238.

ISOZAKI Y, YAO J, Ji Z., SAITOH M., KOBAYASHI N. \& SAKAI H. 2008. - Rapid sea-level change in the Late Guadalupian (Permian) on the Tethyan side of South China: litho- and biostratigraphy of the Chaotian section in Sichuan. Proceeding of Japan Academy, Series B 84: 344-353.

JiN Y., ZHANG J. \& SHAN Q. 1994. - Two phases of the end-Permian mass extinction. In Pangea: global environments and resources. Canadian Society of Petroleum. Memoir 17: 813-822.

JiN Y., ZHANG J. \& SHAN Q. 1995. — Pre-Lopingian catastrophic event of marine fauna. Acta Palaeontologica Sinica 34: 410-427 
Jin Y. G., Shen S. Z., Henderson C. M., Wang X. D., Wang W., Wang Y., CaO, C. Q. \& Shang Q. H. 2006. - The Global Stratotype Section and Point (GSSP) for the boundary between the Capitanian and Wuchiapingian stage (Permian). Episodes 29: 253-262.

Kozur H. W. 1985. - Neue Ostracoden-Arten aus dem oberen Mittelkarbon (Höheres Moskovian) Mittel- und Oberperm des Bükk-Gebirges (N-Ungarn). Geologische-Paläontologische Mitteilungen Innsbruck 2: 1-145.

Lai X., Wang W., Wignall P. B., Bond D. P. G., Jiang H., Ali J. R., John E. H. \& Sun Y. 2008. - Palaeoenvironmental change during the end-Guadalupian (Permian) mass extinction in Sichuan, China. Palaeogeography, Palaeoclimatology, Palaeoecology 269: 78-93.

LeTHIERS F. \& CRASQUin-Soleau S. 1988. - Comment extraire des microfossiles à tests calcitiques de roches calcaires dures. Revue de Micropaléontologie 31: 56-61.

Mei S. L. \& Henderson C. M. 2001. - Evolution of Permian conodont provincialism and its significance in global correlation and paleoclimate implication. Palaeogeography, Palaeoclimatology, Palaeoecology 170: 237-260.

MELNYK D. H. \& MADDOCKS R. F. 1988a. — Ostracode biostratigraphy of the Permo-Carboniferous of central and north-central Texas, Part I: Palaeoenvironmental framework. Micropaleontology 34: 1-20.

MelnyK D. H. \& MAdDOCKS R. F. 1988b. — Ostracode biostratigraphy of the Permo-Carboniferous of central and north-centra Texas, Part II: Ostracode zonation. Micropaleontology 34: 1-40.

Mette W. 2008. - Upper Permian and lowermost Triassic stratigraphy, facies and ostracods in NW Iran - implications for the P/T extinction event. Stratigraphy 5 (2): 205-219.

Moore R. C. 1961. - Treatise on Invertebrate Paleontology. Arthropoda 3, Crustacea, Ostracoda. Geological Society of America and University of Kansas Press, Lawrence, 442 p.

Retallack G. J. \& Jahren A. H. 2008. - Methane release from igneous intrusion of coal during Late Permian extinction events. Journal of Geology 116: 1-20.

Saitoh M., IsozaKi Y., YaO J., Ji, Z., Ueno Y. \& Yoshida N. 2013a. - The appearance of an oxygen-depleted condition on the Capitanian disphotic slope/basin in South China: MiddleUpper Permian stratigraphy at Chaotian in Northern Sichuan. Global and Planetary Change 105: 180-192.

Saitoh M., Isozaki Y., Ueno Y., Yoshida N., YaO J. \& Ji Z. 2013b. - Middle-Upper carbon isotope stratigraphy at Chaotian, South China: Pre-extinction multiple upwelling of oxygendepleted water onto continental shelf. Journal of Asian Earth Sciences, 67-68: 51-62

SCOTESE C. R. \& LANGFord R. P. 1995. — Pangea and the paleogeography of the Permian, in Scholle P. A., PerYT T. M. \& Ulmer-Scholle D. S. (eds), The Permian of Northern Pangaea. Volume 1. Paleogeography, Paleoclimates, Stratigraphy. Springer, Berlin: 3-19.

ShaO, L., Zhang P, Ren D. \& Lei J. 1998. — Late Permian coalbearing carbonate successions in southern China: coal accumulation on carbonate platforms. International Journal of Coal Geology 37: 235-256.

ShaO L., Zhang P., GaYer R. A., Chen J. L. \& DAI S. F. 2003. Coal in a carbonate sequence stratigraphic framework: the Upper Permian Heshan Formation in central Guangxi, southern China. Journal of the Geological Society 160: 285-298.
SHEN S. Z. \& SHI G. R. 2009. — Latest Guadalupian brachiopods from the Guadalupian/Lopingian boundary GSSP section at Penglaitan in Laibin, Guangxi, South China and implications for the timing of the pre-Lopingian crisis. Palaeoworld 18: 152-161.

SHI C. G. \& CHEN D. Q. 1987. — The Changhsingian Ostracodes from Meishan, Chanxing, Zhejiang, in Nanjing Institute of Geology and Palaeontology, Academia Sinica (ed.), Stratigraphy and Palaeontology of Systemic Boundaries in China, Permian-Triassic boundary (1). Nanjing University Press, Nanjing: 23-80.

SHI C. G. \& CHEN D. Q. 2002. — Late Permian Ostracodes from Heshan and Yishan of Guangxi. Bulletin of Nanjing Institute Geology and Paleontology, Academic Sinica 15: 47-129 (in Chinese with English abstract).

Stanley D. J. \& YANG X. 1994. - Double mass-extinction at the end of the Paleozoic era. Science 266: 1340-1344.

TANaKa G., Ono T., Yuan A., Ichida M. \& Maeda H. 2012. Early Permian Ostracods from Mugi County, Gifu Prefecture, Central Japan. Paleontological Research 16: 88-106.

TanaKa G., Ono T., Nishimura T. \& Maeda H. 2013. — Middle Permian ostracods from the Akasaka Limestone,Gifu Prefecture, central Japan. Paleontological Research 16: 289-306.

Thompson G. M., Ali J. R., Song H. \& Jolley D. W. 2001. Emeishan basalts, SW China: reappraisal of the formation's type area stratigraphy and a discussion of its significance as a large igneous province. Journal of the Geological Society 158: 593-599.

Ulrich E. O. \& BASSLER R. S. 1923. — Paleozoic Ostracoda: Their morphology, classification and occurrence. Maryland Geological Survey, Silurian 9: 271-391.

Veizer J., Godderis Y. \& Francois L. M. 2000. — Evidence for decoupling of atmospheric $\mathrm{CO}_{2}$ and global climate during the Phanerozoic eon. Nature 408: 698-701.

WaNG S. Q. 1978. — Late Permian and Early Triassic ostracods of Western Guizhou and northeastern Yunnan. Acta Palaeontologica Sinica 17: 277-312 (in Chinese with English abstract).

Wang X.-D. \& SugrYAma T. 2000. - Diversity and extinction patterns of Permian coral faunas of China. Lethaia 33: 285-294.

WANG Y. \& JIN Y. G. 2000. - Permian palaeogeographic evolution of the Jiangnan Basin, South China. Palaeogeography, Palaeoclimatology, Palaeoecology 160: 35-44.

Wei M., Li Z., JiAng Z. \& Xie L. 1983. — Arthropoda, in Chengdu Institute of Geological and Mineral Resources (ed.), Palaeontological Atlas of Southwest China. Geological Publishing House, Beijing: 7-253.

Wignall P. B., Védrine S., Bond D. P. G., Wang W., Lai X. L., ALI J. R. \& JiAnG H. S. 2009. - Facies analysis and sea-level change at the Guadalupian-Lopingian Global stratotype (Laibin, South China), and its bearing on the end-Guadalupian mass extinction. Journal of the Geological Society 166: 655-666.

Yin H. F., Feng Q., Lai X., Baud A. \& Tong J. 2007. — The protracted Permo-Triassic crisis and multi-episode extinction around the Permian-Triassic boundary. Global and Planetary Change 55: 1-20.

Yuan A., Crasquin S., Feng Q. \& Gu S. 2007. — Latest Permian deep-water ostracods from Southwestern Guangxi, South China. Journal of Micropalaeontology 26: 169-191.

YuAN A., CRasquin S., Feng Q. \& Gu S. 2009. — Ostracods from the uppermost Permian siliceous and muddy rocks of Guizhou, Guangxi and Anhui. Acta Micropalaeontologica Sinica 26: 385-403.

ZhaO J. K., Liang X. L. \& Zheng Z. G. 1978. — Late Permian cephalopods of South China. Palaeontologica Sinica 12: 1-194. 